\title{
Anelasticity across seismic to tidal timescales: a self-consistent approach
}

\section{Citation}

Lau, Harriet C.P., Ulrich Faul, Jerry X. Mitrovica, David Al-Attar, Jeroen Tromp, and Gordana Garapić. 2016. "Anelasticity across Seismic to Tidal Timescales: A Self-Consistent Approach." Geophysical Journal International 208 (1): 368-84. https://doi.org/10.1093/gji/ggw401.

\section{Permanent link}

http://nrs.harvard.edu/urn-3:HUL.InstRepos:41401412

\section{Terms of Use}

This article was downloaded from Harvard University's DASH repository, and is made available under the terms and conditions applicable to Other Posted Material, as set forth at http:// nrs.harvard.edu/urn-3:HUL.InstRepos:dash.current.terms-of-use\#LAA

\section{Share Your Story}

The Harvard community has made this article openly available.

Please share how this access benefits you. Submit a story.

Accessibility 


\title{
Anelasticity across seismic to tidal timescales: a self-consistent approach
}

\author{
Harriet C.P. Lau, ${ }^{1}$ Ulrich Faul, ${ }^{2}$ Jerry X. Mitrovica, ${ }^{1}$ David Al-Attar, ${ }^{3}$ Jeroen Tromp ${ }^{4}$ \\ and Gordana Garapić ${ }^{5}$ \\ ${ }^{1}$ Department of Earth and Planetary Sciences, Harvard University, Cambridge, MA 02143, USA. E-mail: harrietlau@fas.harvard.edu \\ ${ }^{2}$ Department of Earth, Atmospheric and Planetary Sciences, Massachusetts Institute of Technology, Cambridge, MA 02139, USA \\ ${ }^{3}$ Bullard Laboratories, Department of Earth Sciences, University of Cambridge, Cambridge CB3 0EZ, UK \\ ${ }^{4}$ Department of Geosciences and Program in Applied \& Computational Mathematics, Princeton University, Princeton NJ 08544, USA \\ ${ }^{5}$ Geology Department, State University of New York, New Paltz, NY 12561, USA
}

Accepted 2016 October 19. Received 2016 October 17; in original form 2016 May 25

\section{SUMMAR Y}

In a pioneering study, Wahr \& Bergen developed the widely adopted, pseudo-normal mode framework for predicting the impact of anelastic effects on the Earth's body tides. Lau et al. have recently derived an extended normal mode treatment of the problem (as well as a minor variant of the theory known as the direct solution method) that makes full use of theoretical developments in free oscillation seismology spanning the last quarter century and that avoids a series of assumptions and approximations adopted in the traditional theory for predicting anelastic effects. There are two noteworthy differences between these two theories: (1) the traditional theory only considers perturbations to the eigenmodes of an elastic Earth, whereas the new theory augments this set of modes to include the relaxation modes that arise in anelastic behaviour; and (2) the traditional theory approximates the complex perturbation to the tidal Love number as a scaled version of the complex perturbation to the elastic moduli, whereas the new theory computes the full complex perturbation to each eigenmode. In this study, we highlight the above differences using a series of synthetic calculations, and demonstrate that the traditional theory can introduce significant error in predictions of the complex perturbation to the Love numbers due to anelasticity and the related predictions of tidal lag angles. For the simplified Earth models we adopt, the computed lag angles differ by $\sim 20$ per cent. The assumptions in the traditional theory have important implications for previous studies that use model predictions to correct observables for body tide signals or that analyse observations of body tide deformation to infer mantle anelastic structure. Finally, we also highlight the fundamental difference between apparent attenuation (i.e. attenuation inferred from observations or predicted using the above theories) and intrinsic attenuation (i.e. the material property investigated through experiments), where both are often expressed in terms of lag angles or $Q^{-1}$. In particular, we demonstrate the potentially significant (factor of two or more) bias introduced in estimates of $Q^{-1}$ and its frequency dependence in studies that have treated $Q^{-1}$ determined from tidal phase lags or measured experimentally as being equal. The observed or theoretically predicted lag angle (or apparent $Q^{-1}$ ) differs from the intrinsic, material property due to inertia, self-gravity and effects associated with the energy budget. By accounting for these differences we derive, for a special case, an expression that accurately maps apparent attenuation predicted using the extended normal mode formalism of Lau et al. into intrinsic attenuation. The theory allows for more generalized mappings which may be used to robustly connect observations and predictions of tidal lag angles to results from laboratory experiments of mantle materials.

Key words: Tides and planetary waves; Elasticity and anelasticity; Seismic attenuation; Theoretical seismology. 


\section{INTRODUCTION}

The search for an accurate and complete understanding of the frequency domain response of the Earth to an applied forcing is of fundamental importance in efforts to model a wide range of dynamical processes, including seismic wave propagation, ocean (loading) and body tides, polar motion and glacial isostatic adjustment. Dissipation of energy varies with frequency and, from a macroscopic viewpoint, this variability is largely a reflection of the frequency dependence of the viscosity of Earth's bulk materials. In this study, we focus on the anelastic (small strain) portion of Earth's rheological response from long-period seismic to tidal timescales, and we treat this response as time dependent, but completely recoverable (i.e. we do not consider the possibility of a purely viscous component to the response).

Several approaches have been used to investigate how dissipation arises across these timescales. For example, experimental studies recreate relevant stress and temperature conditions in samples across a specified frequency range and focus on grain-scale processes (see e.g. Faul \& Jackson 2015). These studies indicate that Earth materials exhibit the so-called 'high-temperature background' behaviour, whereby attenuation (which is to be interpreted as synonymous with dissipation) may be characterized by a powerlaw frequency dependence. Materials that exhibit such behaviour include metals (e.g. Rivière 2001), ceramics (e.g. MgO, Barnhoorn et al. 2007) and silicates (e.g. Jackson 1993; Gribb \& Cooper 1998). Microphysical models of grain-scale processes predict a grainsize dependence of dissipation due to elastically and diffusionally accommodated grain-boundary sliding (e.g. Raj \& Ashby 1971; Raj 1975; Morris \& Jackson 2009; Lee et al. 2011). These models predict that with decreasing frequency the mildly frequencydependent high-temperature background or absorption band behaviour is followed seamlessly by a transition to viscous behaviour. The frequency and grain-size dependence predicted by these models has been observed experimentally in olivine (Jackson et al. 2002; Jackson \& Faul 2010).

Direct observations of Earth's response to external forcings have also been used to constrain the frequency dependence of dissipation. For example, measurements of the Earth's response to earthquakes, including body waves, surface waves, and normal mode excitation, have all yielded estimates of the Earth's attenuation (or $Q^{-1}$ where $Q$ is the quality factor) structure, though these estimates are generally characterized by significant uncertainty (Resovsky et al. 2005). In regard to seismic frequencies, models adopted within the literature include an absorption band in which $Q^{-1}$ is assumed to be either constant with respect to frequency (Kanamori \& Anderson 1977) or characterized by a power law dependence (Anderson \& Minster 1979; Shito et al. 2004; Lekić et al. 2009). Observations of geodynamic processes with longer periods, such as body tides and the Chandler wobble, have extended existing constraints on dissipation to include timescales of years and decades (e.g. Anderson \& Minster 1979; Ray et al. 2001; Benjamin et al. 2006), although these observations are also characterized by large uncertainty.

Taken together, existing observational constraints suggest that dissipation increases with the period of the forcing, though the exponent of the frequency dependence and the frequency of the onset of the absorption band remain poorly known. This is, in part, due to the large observational uncertainties noted above (Resovsky et al. 2005; Benjamin et al. 2006), but a more fundamental issue is also at play. In particular, a series of assumptions and approximations commonly adopted in analyses of anelastic behaviour at long (i.e. tidal and Chandler wobble) periods (Wahr \& Bergen 1986) are not consistent with the treatment of dissipation in the seismic band (Lau et al. 2015) and obscure differences between intrinsic (material) and apparent (observed) dissipation. A major goal of the present paper is to highlight these issues and explore, in detail, inaccuracies introduced by adopting the traditional theory for modelling the anelastic response of the Earth in the tidal band. As we will demonstrate, these inaccuracies include significant biases introduced in previous estimates of intrinsic dissipation across tidal timescales and in extrapolating the frequency dependence of dissipation to longer periods.

A rigorous methodology for mapping between sample (i.e. experimental) scale and planetary-scale information, and for bridging between observations at seismic and tidal timescales requires a generalized, self-consistent theoretical treatment of anelasticity. In this paper, we apply, for this purpose, the generalized, normal mode theory of Lau et al. (2015) and a variant of this method [the direct solution (DS) method] that has advantages in the practical application of the theory. In the next section, we begin by summarizing the relevant aspects of this new theory and the traditional approach for modelling anelastic effects in the tidal band (Wahr \& Bergen 1986).

Finally, although we focus here on applications to geophysics, the methodology we develop is equally relevant to studies of other terrestrial planets. We return to this point in the concluding section of the paper.

\section{THEORETICAL BACKGROUND}

Gilbert (1971) highlighted the fundamental connection between the deformation of the Earth in response to earthquakes and luni-solar tidal forcing by pointing out that the normal modes of the Earth system relevant to seismic and tidal applications are precisely the same. In the years subsequent to his seminal study, insights from a series of important developments in seismic free oscillation theory (e.g. Dahlen 1968; Gilbert 1971; Woodhouse \& Dahlen 1978; Woodhouse 1980; Park \& Gilbert 1986) were incorporated into analyses of Earth's body tides and other longer period geodynamic processes (e.g. Smith \& Dahlen 1981; Wahr 1981a,b; Wahr \& Bergen 1986). However, the latter effort did not always mirror the former, as is clear by comparing seismic normal mode treatments and body tide studies of the impact of rotation (compare Dahlen (1968) with Wahr (1981b), Dehant (1987) and Wang (1994)) and asphericity (compare Woodhouse (1980) with Dehant et al. (1999), Métivier \& Conrad (2008), Latychev et al. (2009) and Qin et al. (2014)). Moreover, in considering anelastic effects, the standard perturbation approach adopted in the body tide literature (Wahr \& Bergen 1986) predates the seismic normal mode theories of Lognonné (1991) and Tromp \& Dahlen (1990), who derived generalized expressions for the orthogonality of eigenfunctions appropriate to this case. 
Lau et al. (2015), motivated by the availability of progressively more accurate and extensive space-geodetic measurements of body tide deformation (e.g. Yuan \& Chao 2012; Yuan et al. 2013), derived a generalized normal mode treatment of body tides using the full theoretical machinery of modern seismic free oscillation theory and made appropriate extensions relevant for the tidal application. We begin by reviewing this theory which hereafter we will term the 'extended normal mode' (ENM) theory.

\subsection{Parametrizing anelasticity}

We begin by introducing the frequency-domain fourth-order tensor $\boldsymbol{\Lambda}$, which relates the deformation to the incremental first Piola-Kirchoff stress tensor, $\mathbf{T}^{\mathrm{PK} 1}$, via the following expression:

$\mathbf{T}^{\mathrm{PK} 1}(\omega)=\boldsymbol{\Lambda}(\omega): \nabla \mathbf{s}(\omega)$

Here, $\omega$ is the (complex) frequency and $\mathbf{s}$ is the displacement, $\boldsymbol{\nabla}$ is the del-operator (and thus $\nabla \mathbf{s}$ represents the full deformation tensor) and the double-dot operation represents $\mathbf{A}: \mathbf{B}=\operatorname{tr}\left(\mathbf{A}^{\mathrm{T}} \cdot \mathbf{B}\right)$. The Fourier transform convention for a function $f$ used throughout this study is

$\mathcal{F}[f(t)] \equiv \tilde{f}(\omega)=\int_{0}^{\infty} f(t) e^{-i \omega t} \mathrm{~d} t$

$\mathcal{F}^{-1}[\tilde{f}(\omega)]=f(t)=\frac{1}{2 \pi} \int_{-\infty}^{+\infty} \tilde{f}(\omega) e^{i \omega t} \mathrm{~d} \omega$

$\boldsymbol{\Lambda}$ must be analytic in the lower half of the complex plane (Dahlen \& Tromp 1998) and additionally adhere to the following relations:

$\boldsymbol{\Lambda}^{*}(\omega)=\boldsymbol{\Lambda}\left(-\omega^{*}\right)$

$\Lambda_{i j m n}(\omega)=\Lambda_{m n i j}(\omega)$

where eq. (4) must be satisfied in order to obtain a real-valued $\boldsymbol{\Lambda}$ in the time domain (O'Connell \& Budiansky 1978) and the symmetry relation (5) is commonly assumed to hold within viscoelasticity. For a discussion of the physical significance of eq. (5), see Day (1971a,b). The scalar components of $\boldsymbol{\Lambda}$ represent specific moduli. For an isotropic medium, there are two such moduli: the shear modulus and the bulk modulus, $\mu$ and $\kappa$, respectively. In this specific case, the elements of $\boldsymbol{\Lambda}$ are given by:

$$
\begin{aligned}
\Lambda_{i j m n}(\omega)= & {\left[\kappa(\omega)-\frac{2}{3} \mu(\omega)\right] \delta_{i j} \delta_{m n}+\mu(\omega)\left[\delta_{i m} \delta_{j n}+\delta_{i n} \delta_{j m}\right]+\frac{1}{2}\left(T^{0}{ }_{i j} \delta_{m n}+T^{0}{ }_{m n} \delta_{i j}+T^{0}{ }_{i m} \delta_{j n}-T^{0}{ }_{j m} \delta_{i n}\right.} \\
& \left.-T^{0}{ }_{i n} \delta_{j m}-T^{0}{ }_{j n} \delta_{j m}-T^{0}{ }_{j n} \delta_{i m}\right)
\end{aligned}
$$

(eq. 3.146 and table 3.2 in Dahlen \& Tromp 1998). Here, $\delta_{i j}$ is the Kronecker delta and $\mathbf{T}^{0}$ is the initial stress tensor. Readers are directed to chapters 2-3 of Dahlen \& Tromp (1998) for a comprehensive review of these relations.

For an elastic medium, $\boldsymbol{\Lambda}$ is purely real and independent of $\omega$. (In the following, and if necessary for clarity, we will denote any parameter, $X$, associated with the elastic problem as $\bar{X}$.) If we denote a general scalar elastic modulus by $\bar{M}$, then once anelasticity is introduced

$\bar{M} \rightarrow M(\omega)=\bar{M}+\delta M(\omega)$

where $\delta M$ is complex and a function of the complex frequency, $\omega$, and thus $M(\omega)=M_{1}(\omega)+i M_{2}(\omega)$ where $i=\sqrt{-1}$. We do not discuss the specific frequency dependence of $M(\omega)$ here, though this is an active area of research (e.g. Gribb \& Cooper 1998; Jackson \& Faul 2010; McCarthy et al. 2011; Bellis \& Holtzman 2014). It has been shown experimentally for materials exhibiting absorption band behaviour that $M_{1}(\omega)$ will decrease with decreasing frequency and $M_{2}(\omega)$ will increase (e.g. Faul \& Jackson 2015). The following ratio

$Q(\omega) \equiv \frac{M_{1}(\omega)}{M_{2}(\omega)}$

is known as the quality factor of the modulus $M$ (Chapter 1.3 in Nowick \& Berry 1972), where $\arcsin \left[Q^{-1}(\omega)\right]$ describes the lag angle between the stress and strain acting on $M$, and is also known as the loss angle. We note that in many applications where $Q \gg 1$ (and thus the lag angle is small), this angle is often listed as $\arctan \left[Q^{-1}(\omega)\right]$. For a more complete discussion of this issue, the reader is referred to Efroimsky (2012b). In this study, which is limited to the high $Q$ regime, we will adopt the approximate form to aid comparisons to the literature. We emphasize, however, that our theory does not require such an approximation. $Q$ may also be interpreted in terms of energy loss where $Q^{-1}$ represents the fractional average energy dissipated per cycle and so we may also write

$Q^{-1} \equiv \frac{\oint \dot{E} \mathrm{~d} t}{2 \omega \oint E \mathrm{~d} t}$

where $E$ is the elastic energy density (Chapter 61.6 in Dahlen \& Tromp 1998). The numerator and denominator represent the dissipated and stored energies per cycle, respectively. The interpretation of $Q^{-1}$ has caused confusion in the past, as outlined by O'Connell \& Budiansky (1978) and in this study we will highlight some continuing misinterpretations in the tidal application of $Q$ - a key parameter that is ubiquitous in discussions of anelasticity-in Section 3.2. 
To summarize, all information concerning the anelastic properties of the medium is stored within $\boldsymbol{\Lambda}(\omega)$. How $\boldsymbol{\Lambda}(\omega)$ fits into the general framework of long period seismic and body tide problems is addressed next.

\subsection{An ENM theory}

Consider the governing equation for a self-gravitating, non-rotating, elastic Earth, forced by some arbitrary body force, $\tilde{\mathbf{f}}$. The frequencydomain equation in this case is given by

$\overline{\mathcal{H}}(\mathbf{r}) \mathbf{s}(\mathbf{r} ; \omega)-\omega^{2} \mathbf{s}(\mathbf{r} ; \omega)=\tilde{\mathbf{f}}(\mathbf{r} ; \omega)$,

where

$\rho(\mathbf{r}) \overline{\mathcal{H}}(\mathbf{r}) \mathbf{s}(\mathbf{r} ; \omega)=\rho(\mathbf{r}) \nabla \phi(\mathbf{r} ; \omega)+\rho(\mathbf{r}) \mathbf{s}(\mathbf{r} ; \omega) \cdot \nabla \nabla(\Phi(\mathbf{r}))-\nabla \cdot[\overline{\mathbf{\Lambda}}(\mathbf{r}): \nabla \mathbf{s}(\mathbf{r} ; \omega)]$.

Here, $\mathbf{r}$ is the position vector whose origin is at the centre of mass (we will use this to denote radius, $r$, co-latitude, $\theta$, and east-longitude, $\psi$ ), $\rho$ is the mass density, and the following gravitational quantities,

$\Phi(\mathbf{r})=-G \int_{V} \frac{\rho\left(\mathbf{r}^{\prime}\right)}{\left\|\mathbf{r}-\mathbf{r}^{\prime}\right\|} \mathrm{d} V^{\prime}$,

$\phi(\mathbf{r} ; \omega)=-G \int_{V} \frac{\rho\left(\mathbf{r}^{\prime}\right) \mathbf{s}\left(\mathbf{r}^{\prime}, \omega\right) \cdot\left(\mathbf{r}-\mathbf{r}^{\prime}\right)}{\left\|\mathbf{r}-\mathbf{r}^{\prime}\right\|^{3}} \mathrm{~d} V^{\prime}$,

are the equilibrium gravitational potential and incremental gravitational potential, respectively. In these expressions, $G$ is the universal gravitational constant and $V$ is a volume that encompasses the Earth. We have omitted the impact of rotation on these expressions in order to simplify discussion, however, the theory described in Lau et al. (2015) is able to incorporate this effect. Eq. (10) is solved along with the following traction-free boundary condition:

$\hat{\mathbf{n}} \cdot[\overline{\mathbf{\Lambda}}(\mathbf{r}): \nabla \mathbf{s}(\mathbf{r} ; \omega)]=\mathbf{0}, \quad \mathbf{r} \in \Omega$,

where $\Omega$ is the surface of the Earth with unit outward normal $\hat{\mathbf{n}}$. When anelasticity is introduced $\bar{\Lambda}(\mathbf{r}) \rightarrow \boldsymbol{\Lambda}(\mathbf{r} ; \omega)$ and the governing equation (10) becomes

$\mathcal{H}(\mathbf{r} ; \omega) \mathbf{s}(\mathbf{r} ; \omega)-\omega^{2} \mathbf{s}(\mathbf{r} ; \omega)=\tilde{\mathbf{f}}(\mathbf{r} ; \omega)$

In the following, we will treat any dependency on $\mathbf{r}$ and $\omega$ as implicit unless the clarity of meaning demands otherwise. The homogeneous version of eq. (15) is

$\mathcal{H}\left(\omega_{k}\right) \mathbf{s}_{k}-\omega_{k}^{2} \mathbf{s}_{k}=\mathbf{0}$

where $\mathbf{s}_{k}$ and $\omega_{k}$ are the eigenfunctions and eigenfrequencies, respectively, for which there exists a non-trivial solution to eq. (16). (Note the non-linearity in the eigenvalue parameter.) In Lau et al. (2015), the duality product first derived by Lognonné (1991) was incorporated into the theory. In this paper, we choose to instead adopt the inner product to aid the comparison between the ENM theory and the traditional normal mode theory of Wahr \& Bergen (1986). (Yang \& Tromp (2015) showed that the duality and inner product lead to equivalent results.) Following Yang \& Tromp (2015), we define the inner product as

$\left\langle\mathbf{s}_{k}, \mathbf{s}_{k^{\prime}}\right\rangle=\int_{V} \rho \mathbf{s}_{k}^{*} \cdot \mathbf{s}_{k^{\prime}} \mathrm{d} V$

which has the following symmetries:

$\left\langle\mathbf{s}_{k}, \mathbf{s}_{k^{\prime}}\right\rangle=\left\langle\mathbf{s}_{k^{\prime}}, \mathbf{s}_{k}\right\rangle^{*}$,

$\left\langle\mathbf{s}_{k}, \mathcal{H}\left(\omega_{k^{\prime}}\right) \mathbf{s}_{k^{\prime}}\right\rangle=\left\langle\mathcal{H}^{*}\left(\omega_{k}\right) \mathbf{s}_{k}, \mathbf{s}_{k^{\prime}}\right\rangle=\left\langle\mathbf{s}_{k^{\prime}}, \mathcal{H}^{*}\left(\omega_{k}\right) \mathbf{s}_{k}\right\rangle^{*}$.

The eigenfunctions are normalized as follows

$\left\langle\mathbf{s}_{k}, \mathbf{s}_{k^{\prime}}\right\rangle-\frac{1}{2} \omega_{k}^{-1}\left\langle\mathbf{s}_{k}, \partial_{\omega} \mathcal{H}\left(\omega_{k^{\prime}}\right) \mathbf{s}_{k^{\prime}}\right\rangle=\delta_{k k^{\prime}}$.

Thus, eq. (16) has the following symmetry

$\mathcal{H}\left(-\omega^{*}\right)=\mathcal{H}^{*}(\omega)$

and as such, one finds that if and only if $\omega_{k}$ and $\mathbf{s}_{k}$ are eigenmodes of eq. (16), then so too are $-\omega^{*}$ and $\mathbf{s}^{*}$ (Lognonné 1991 ).

After finding the eigenmodes, any solution $\mathbf{s}$ may be formed by the appropriate weighted sum of eigenfunctions $\mathbf{s}_{k}$ with time dependence given by $\omega_{k}$. In normal mode seismology, a Green function for such a system, that is the solution to a point force (subject to the appropriate initial and boundary conditions), is given by (Lognonné 1991)

$\mathbf{G}\left(\mathbf{r}, \mathbf{r}^{\prime} ; t\right)=\operatorname{Re} \sum_{k} \frac{1}{2 i \omega_{k}} \mathbf{s}_{k}(\mathbf{r}) \mathbf{s}_{k}{ }^{*}\left(\mathbf{r}^{\prime}\right) \exp \left(i \omega_{k} t\right)$. 
The function $\mathbf{G}$ is simplified in the normal mode seismology application due to the symmetry of eigenfrequencies $\omega_{k}$ and $-\omega_{k}{ }^{*}$ such that $\mathbf{G}$ is often quoted as (e.g. Dahlen \& Tromp 1998):

$\mathbf{G}\left(\mathbf{r}, \mathbf{r}^{\prime} ; t\right)=\operatorname{Re} \sum_{\operatorname{Re}_{\left\{\omega_{k}\right\rangle>0}} \frac{1}{i \omega_{k}} \mathbf{s}_{k}(\mathbf{r}) \mathbf{s}_{k}^{*}\left(\mathbf{r}^{\prime}\right) \exp \left(i \omega_{k} t\right)$,

where the summation includes only eigenfrequencies where $\operatorname{Re}\left\{\omega_{k}\right\}>0$. The simplification implied by eq. (23) cannot be used in the tidal approach due to the existence of 'relaxation modes' which will be discussed in Section 2.2.1 (or see Lau et al. 2015). G describes the time-domain response to an impulse force and convolving $\mathbf{G}$ with a time-domain force, $\mathbf{f}$, yields the solution, $\mathbf{s}$,

$$
\begin{aligned}
\mathbf{s}(\mathbf{r} ; t)= & \int_{t_{0}}^{t} \int_{V} \mathbf{G}\left(\mathbf{r}, \mathbf{r}^{\prime} ; t-t^{\prime}\right) \cdot \mathbf{f}\left(\mathbf{r}^{\prime}, t^{\prime}\right) \mathrm{d} V^{\prime} \mathrm{d} t^{\prime}+\int_{t_{0}}^{t} \int_{\Omega} \mathbf{G}\left(\mathbf{r}, \mathbf{r}^{\prime} ; t-t^{\prime}\right) \cdot \boldsymbol{\tau}\left(\mathbf{r}^{\prime}, t^{\prime}\right) \mathrm{d} \Omega^{\prime} \mathrm{d} t^{\prime}+\int_{t_{0}}^{t} \int_{V} \mathbf{B}\left(\mathbf{r}, \mathbf{r}^{\prime} ; t-t^{\prime}\right) \cdot \mathbf{f}\left(\mathbf{r}^{\prime}, t^{\prime}\right) \mathrm{d} V^{\prime} \mathrm{d} t^{\prime} \\
& +\int_{t_{0}}^{t} \int_{\Omega} \mathbf{B}\left(\mathbf{r}, \mathbf{r}^{\prime} ; t-t^{\prime}\right) \cdot \boldsymbol{\tau}\left(\mathbf{r}^{\prime}, t^{\prime}\right) \mathrm{d} \Omega^{\prime} \mathrm{d} t^{\prime},
\end{aligned}
$$

where $t_{0}$ is the time of initiation of forcing $\mathbf{f}$. The expression (24) includes an additional traction, $\boldsymbol{\tau}$, which generalizes our application to include any surface force. $\mathbf{B}(\mathbf{r} ; t)$ is the contribution due to singularities of the frequency domain solution other than isolated simple poles, which could potentially include features like branch cuts or other non-modal singularities. $\mathbf{B}$ arises due to the specific form we will adopt for $\boldsymbol{\Lambda}(\omega)$ (see Section 2.2.1). The existence of these non-modal singularities within the complex plane of $\omega$ greatly complicates the evaluation of eq. (24).

We will consider the form of $\mathbf{s}(\mathbf{r} ; t)$ for two cases: the long period response to an earthquake with body force $\mathbf{f}$ and traction $\boldsymbol{\tau}$; and the body tide response to the forcing $\rho \nabla \Psi$ (where $\Psi$ is the tidal potential). In the earthquake problem, this form is written as

$\mathbf{s}(\mathbf{r} ; t)=\int_{t_{0}}^{t} \int_{V} \mathbf{G}\left(\mathbf{r}, \mathbf{r}^{\prime} ; t-t^{\prime}\right) \cdot \mathbf{f}\left(\mathbf{r}^{\prime}, t^{\prime}\right) \mathrm{d} V^{\prime} \mathrm{d} t^{\prime}+\int_{t_{0}}^{t} \int_{\Omega} \mathbf{G}\left(\mathbf{r}, \mathbf{r}^{\prime} ; t-t^{\prime}\right) \cdot \boldsymbol{\tau}\left(\mathbf{r}^{\prime}, t^{\prime}\right) \mathrm{d} \Omega^{\prime} \mathrm{d} t^{\prime}$,

where $\int_{t_{0}}^{t} \int_{V} \mathbf{B}\left(\mathbf{r}, \mathbf{r}^{\prime} ; t-t^{\prime}\right) \cdot \mathbf{f}\left(\mathbf{r}^{\prime}, t^{\prime}\right) \mathrm{d} V^{\prime} \mathrm{d} t^{\prime} \approx 0$ and $\int_{t_{0}}^{t} \int_{\Omega} \mathbf{B}\left(\mathbf{r}, \mathbf{r}^{\prime} ; t-t^{\prime}\right) \cdot \boldsymbol{\tau}\left(\mathbf{r}^{\prime}, t^{\prime}\right) \mathrm{d} \Omega^{\prime} \mathrm{d} t^{\prime} \approx 0$. These approximations are appropriate for many seismic applications since, for plausible forms of $\boldsymbol{\Lambda}$, seismic waves do not occur at very low frequencies and thus do not excite the non-modal singularities associated with $\mathbf{B}$ to an appreciable extent. However, $\mathbf{B}$ is likely to be relevant for post-seismic deformation which occurs at lower frequencies than co-seismic deformation. In contrast, for the body tide, the appropriate expression is

$\mathbf{s}(\mathbf{r} ; t)=\int_{t_{0}}^{t} \int_{V} \rho\left(\mathbf{r}^{\prime}\right) \mathbf{G}\left(\mathbf{r}, \mathbf{r}^{\prime} ; t-t^{\prime}\right) \cdot \nabla \Psi\left(\mathbf{r}^{\prime}, t^{\prime}\right) \mathrm{d} V^{\prime} \mathrm{d} t^{\prime}+\int_{t_{0}}^{t} \int_{V} \rho\left(\mathbf{r}^{\prime}\right) \mathbf{B}\left(\mathbf{r}, \mathbf{r}^{\prime} ; t-t^{\prime}\right) \cdot \nabla \Psi\left(\mathbf{r}^{\prime}, t^{\prime}\right) \mathrm{d} V^{\prime} \mathrm{d} t^{\prime}$,

where one may include in eq. (26) the surface forcing in the case of ocean tidal loading by including the terms on the second and fourth lines of eq. (24) and the appropriate expression for $\boldsymbol{\tau}$. In this case, realistic periodicities of the exciting potential $\nabla \Psi$ are long enough for the contribution from $\mathbf{B}$ to be relevant.

For the seismic problem, the full time dependence of the response is of interest, but for the tidal problem the steady-state solution is required (that is, the transient component of the solution to eq. (26) associated with the initiation of forcing may be ignored). If we assume $\Psi(\mathbf{r} ; t)$ has the form of a single harmonic we may write:

$\nabla \Psi_{\ell m}(\mathbf{r} ; t)=c_{\ell m} \exp \left[i \omega_{T} t\right] \frac{r^{\ell-1}}{a^{\ell}}\left[\ell Y_{\ell m}(\theta, \psi) \hat{\mathbf{r}}+\sqrt{\ell(\ell+1)} \nabla_{1} Y_{\ell m}(\theta, \psi)\right]$

where $a$ is the radius of the Earth, $\omega_{T}$ is the (real) tidal frequency, and $c_{\ell m}$ is a factor that accounts for the orbital properties of the forcing body which produces $\Psi_{\ell m} . Y_{\ell m}$ is the spherical harmonic of degree $\ell$ and order $m$ following the normalization of Edmonds (1960). $\nabla_{1}$ represents the projection of the gradient operator in the $\theta$ and $\psi$ directions. In reality, there are many forcing harmonics but the linearity of the problem allows the suite of tidal harmonics to be trivially superimposed.

At this point we depart from the treatment of a generalized Earth model to focus on the specialized case of a spherically symmetric Earth model. We do this to take advantage of analytic results that will be useful in the illustrative case studies described in Section 3 . The theory of Lau et al. (2015) may be used to incorporate aspherical structure into any of those case studies. For spherically symmetric (and self-gravitating) Earth models, the forcing given by eq. (27) will excite only spheroidal eigenmodes, $\mathbf{s}_{k}$, for which the eigenfunctions for these may be expressed as

${ }_{n} \mathbf{s}_{\ell m}(\mathbf{r})={ }_{n} U_{\ell}(r) Y_{\ell m}(\theta, \psi) \hat{\mathbf{r}}+{ }_{n} V_{\ell}(r) \frac{\nabla_{1} Y_{\ell m}(\theta, \psi)}{\sqrt{\ell(\ell+1)}}$.

The single index $k$ is used to denote $\ell, m$ and the overtone number, $n$, of a mode. Note that we have dropped the $m$ subscript for eigenfunctions ${ }_{n} U_{\ell}$ and ${ }_{n} V_{\ell}$ as spherical symmetry results in degenerate eigenmodes in $m$. When necessary, $\ell, m$ and $n$ will be specified separately.

Thus, by substituting eqs (27) and (28) into eq. (26), and taking the limit $t_{0} \rightarrow-\infty$, the steady-state solution is given by

$\mathbf{s}(\mathbf{r}, t)=-\sum_{n} \frac{{ }_{n} \mathbf{s}_{\ell m}(\mathbf{r})}{2_{n} \omega_{\ell}{ }^{2}} \int_{V} \rho\left(r^{\prime}\right)_{n} \mathbf{s}_{\ell m}{ }^{*}\left(\mathbf{r}^{\prime}\right) \cdot \nabla \Psi_{\ell m}(\mathbf{r} ; t) \mathrm{d} V^{\prime}-\int_{-\infty}^{t} \rho\left(r^{\prime}\right) \mathbf{B}\left(\mathbf{r}, \mathbf{r}^{\prime} ; t-t^{\prime}\right) \cdot \nabla \Psi_{\ell m}\left(\mathbf{r}^{\prime} ; t^{\prime}\right) \mathrm{d} V^{\prime} \mathrm{d} t^{\prime}$. 
After some algebra (which is detailed in Appendix A), one finds that

$$
\begin{aligned}
\sum_{n}{ }_{n} \mathbf{s}_{\ell m}(\mathbf{r}) \int_{V} \rho\left(r^{\prime}\right){ }_{n} \mathbf{s}_{\ell m}{ }^{*}\left(\mathbf{r}^{\prime}\right) \cdot \nabla \Psi_{\ell m}(\mathbf{r} ; t) \mathrm{d} V^{\prime}= & \left\{\sum_{n}{ }_{n} U_{\ell}(r) \int_{0}^{a} \rho(r) \frac{r^{\ell+1}}{a^{\ell}}\left[\ell_{n} U_{\ell}(r)+\sqrt{\ell(\ell+1)_{n}} V_{\ell}(r)\right]\right. \\
& \times \mathrm{d} r \Psi_{\ell m}(\mathbf{r}) \hat{\mathbf{r}}+\sum_{n} \frac{{ }_{n} V_{\ell}(r)}{\sqrt{\ell(\ell+1)}} \int_{0}^{a} \rho(r) \frac{r^{\ell+1}}{a^{\ell}}\left[\ell_{n} U_{\ell}(r)\right. \\
& \left.\left.+\sqrt{\ell(\ell+1)_{n}} V_{\ell}\right] \mathrm{d} r \nabla_{1} \Psi_{\ell m}(\mathbf{r})\right\} \exp \left[i \omega_{T} t\right] .
\end{aligned}
$$

This term represents the excitation of spheroidal modes of degree $\ell$ by a tidal potential of the same degree, $\ell$.

\subsubsection{Eigenfrequencies on the complex plane}

Complex eigenfrequencies incorporate two types of behaviour: oscillation $\left(\operatorname{Re}\left\{\omega_{k}\right\}\right)$ and relaxation $\left(\operatorname{Im}\left\{\omega_{k}\right\}, w h e r e \operatorname{Im}\left\{\omega_{k}\right\}>0\right)$. $\operatorname{These}$ behaviours may be represented on the complex plane of $\omega$, where the set of $\omega_{k}$ are singularities of $\tilde{\mathbf{G}}\left(\mathbf{r}, \mathbf{r}^{\prime} ; \omega\right)$ (the Fourier transform of the Green tensor, G). In seismic free oscillation theory, the eigenfrequencies lie just above the real line, that is, $\operatorname{Im}\{\omega\} \ll \operatorname{Re}\{\omega\}$, and we will henceforth refer to these as 'dynamic modes'. Fig. 1(a) provides a schematic representation of the location of these dynamic modes on the $\omega$-plane. Due to the symmetry property stated in Section 2.2, these eigenfrequencies are comprised of reflected pairs about the imaginary axis. In seismic normal mode calculations, it is common to assume that these modes 'see' Earth structure at real eigenfrequencies, since the imaginary components are so small (Dahlen \& Tromp 1998). To incorporate the effects of anelasticity on the eigenfrequency, a perturbation theory is used that determines the deviation from the elastic eigenfrequency by taking into account the $Q$ structure of the Earth. In general, we have

$\omega_{k} \approx \bar{\omega}_{k}+\delta \omega_{k}$

where $\delta \omega_{k}$ is the small complex perturbation moving $\bar{\omega}$ (an eigenfrequency computed for an elastic Earth) upward and laterally in Fig. 1(a). The real perturbation is due to dispersion (i.e. frequency dependence) and the imaginary perturbation is due to attenuation, both a consequence

(a)

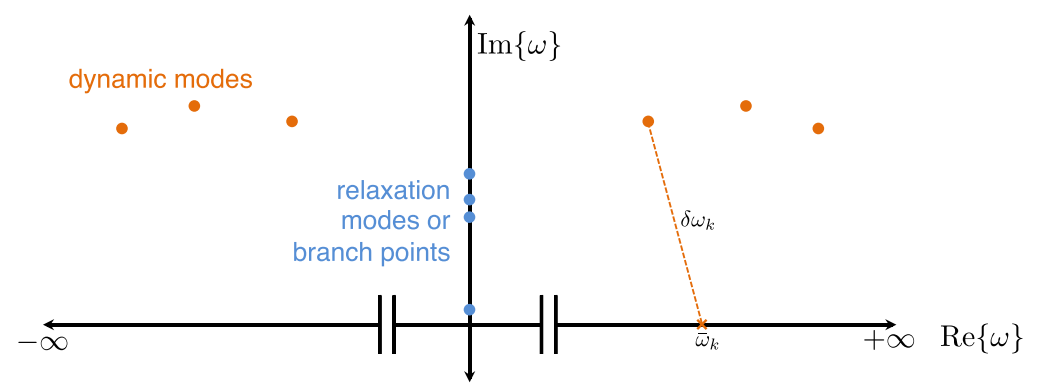

(b)

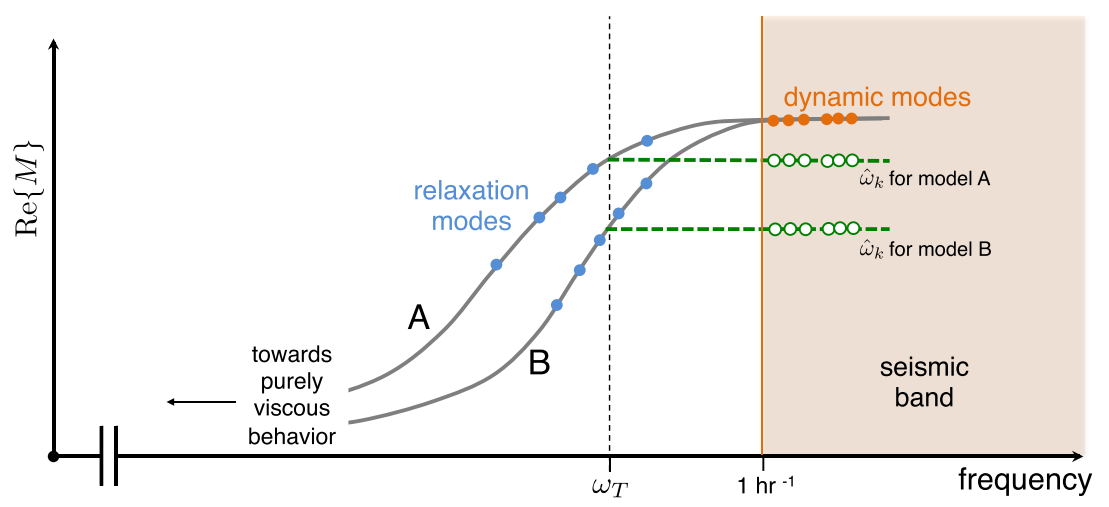

Figure 1. (a) A schematic diagram of the complex plane of $\omega$. Orange circles mark the dynamic modes used in seismic normal mode theory. Blue circles mark the position of relaxation modes required, in addition to the dynamic modes, to accurately express the response to a tidal potential forcing. The orange dashed line labelled $\delta \omega_{k}$ is the complex perturbation from the elastic eigenfrequency, $\bar{\omega}_{k}$, due to anelastic effects. (b) A schematic diagram of the (real) modulus reduction for two materials, $\mathrm{A}$ and $\mathrm{B}$, due to the introduction of anelasticity. As in (a), orange circles mark the dynamic modes and blue circles mark the (distinct) relaxation modes associated with the two models. Green circles illustrate the softening of the modulus assumed in the TNM treatment of anelastic effects on body tides (see the text). 
of introducing anelasticity. The summation within the expression for $\mathbf{G}\left(\mathbf{r}, \mathbf{r}^{\prime} ; t\right)$ for the time-domain Green tensor (eq. 22) can be equivalently expressed as an inversion of $\tilde{\mathbf{G}}\left(\mathbf{r}, \mathbf{r}^{\prime} ; \omega\right)$ based on Cauchy's residue theorem, where one assumes that singularities in the $\omega$-plane are isolated simple poles (Al-Attar 2007).

Lau et al. (2015) demonstrated that for the tidal problem additional contributions must be incorporated into the summation in eq. (22). These so-called 'relaxation' modes would be located along the imaginary axis (i.e. $\operatorname{Re}\left\{\omega_{k}\right\}=0$ ) with a magnitude that is much smaller than the imaginary part of the dynamic modes (Fig. 1a). Calculations in Lau et al. (2015) yielded a factor of $\sim 10$ difference in magnitudes, however this factor is highly model-dependent. These modes also exist in the seismic application but are, in most cases, of negligible importance in that frequency band. Relaxation modes relevant to the post-glacial rebound modes would exist at smaller values along the positive imaginary axis (that is, relatively long relaxation times) provided that such behaviour is incorporated in $\boldsymbol{\Lambda}(\omega)$.

For models of $\boldsymbol{\Lambda}(\omega)$ proposed in the literature (e.g. Anderson \& Minster 1979; Benjamin et al. 2006; Faul \& Jackson 2015) branch points exist on the imaginary axis, and avoiding these features by taking branch cuts leads to the additional term involving the function $\mathbf{B}$ in eq. (26). The structure of $\mathbf{B}$ is often too complicated to express analytically and in the following sections we will depart from the ENM methodology and adopt a slight variant of this method that avoids these complexities. Nevertheless, the above derivation highlights: (1) the importance of augmenting the mode set that seismologists consider with relaxation modes to rigorously capture the tidal response; and (2) the deep connection between the seismic and tidal applications of normal mode theory.

\subsubsection{DS method}

The ENM treatment of body tides is useful for building physical intuition since eigenfrequencies reflect the characteristic timescales of the response (in the case of both the dynamic and relaxation modes) while the sensitivity to internal structure is given by the depth-variation of eigenfunctions. However, as noted above, alternative methods for the tidal problem are necessary once more realistic models of $\boldsymbol{\Lambda}(\omega)$ are adopted. One such method, which in its practical implementation has close similarities to the ENM approach, is the so-called DS method which has been applied in seismological applications (e.g. Hara et al. 1993; Al-Attar 2007; Al-Attar et al. 2012). Rather than finding the eigenmodes of the system (which by definition satisfy eq. 16), the DS method solves the equations governing the forced system (eq. 15).

Following Al-Attar (2007), we express the solution s as a superposition of an appropriate set of basis functions which, for example, may be eigenfunctions of an elastic Earth, $\overline{\mathbf{s}}$. That is, the solutions to the equation

$\overline{\mathcal{H}} \overline{\mathbf{s}}_{k}-\bar{\omega}_{k}^{2} \overline{\mathbf{s}}_{k}=\mathbf{0}$.

Thus, we seek appropriate values of $\beta$ such that

$\mathbf{s}(\mathbf{r} ; t)=\sum_{k} \beta_{k}(t) \overline{\mathbf{s}}_{k}(\mathbf{r})$.

Substituting eq. (33) into eq. (15) and using eq. (27) for the forcing term $b \hat{b m} f$ yields

$\sum_{k^{\prime}}\left(\left\langle\overline{\mathbf{s}}_{k}, \mathcal{H}\left(\omega_{T}\right) \overline{\mathbf{s}}_{k^{\prime}}\right\rangle-\omega_{T}^{2}\left\langle\overline{\mathbf{s}}_{k}, \overline{\mathbf{s}}_{k^{\prime}}\right\rangle\right) \beta_{k^{\prime}}=-\left\langle\overline{\mathbf{s}}_{k}, \nabla \tilde{\Psi}\right\rangle$,

where the normalization for an elastic earth is $\left\langle\overline{\mathbf{s}}_{k}, \overline{\mathbf{s}}_{k^{\prime}}\right\rangle=\delta_{k k^{\prime}}$. $\tilde{\Psi}$ is the frequency-domain forcing where $\Psi(t)=\tilde{\Psi} \exp \left[i \omega_{T} t\right]$. The second term $\left\langle\overline{\mathbf{s}}_{k}, \mathcal{H}\left(\omega_{T}\right) \overline{\mathbf{s}}_{k^{\prime}}\right\rangle$ introduces anelastic coupling between the elastic eigenfunctions; that is, non-zero elements exist when $k \neq k^{\prime}$. It may be shown that

$\left\langle\overline{\mathbf{s}}_{k}, \mathcal{H}\left(\omega_{T}\right) \overline{\mathbf{s}}_{k^{\prime}}\right\rangle=\bar{\omega}_{k^{\prime}}{ }^{2} \delta_{k^{\prime} k}+\left\langle\overline{\mathbf{s}}_{k}, \delta \mathcal{H}\left(\omega_{T}\right) \overline{\mathbf{s}}_{k^{\prime}}\right\rangle$

where $\delta \mathcal{H}\left(\omega_{T}\right)$ acts to perturb $\overline{\mathcal{H}}$ (associated with the elastic problem) to the value required by the anelastic $\boldsymbol{\Lambda}\left(\omega_{T}\right)$. Lau et al. (2015) showed that for aspherical Earth models full coupling is essential to accurately capture any prediction of tidal deformation. However, in the special case of spherically symmetry we are considering here they demonstrated that self-coupling (which we define as including diagonal components only) provides an accurate approximation for sensible models of $\boldsymbol{\Lambda}\left(\omega_{T}\right)$. Adopting the self-coupling approximation, we find that

$\beta_{k}=\frac{\left\langle\overline{\mathbf{s}}_{k}, \nabla \tilde{\Psi}\right\rangle}{\bar{\omega}_{k}^{2}-\omega_{T}^{2}+\left\langle\overline{\mathbf{s}}_{k}, \delta \mathcal{H}\left(\omega_{T}\right) \overline{\mathbf{s}}_{k}\right\rangle}$.

Thus, the steady-state solution is given by

$\mathbf{s}(t)=\sum_{k} \frac{\int_{V} \rho\left(\mathbf{r}^{\prime}\right) \overline{\mathbf{s}}_{n}{ }^{*} \cdot \nabla \tilde{\Psi} \mathrm{d} V^{\prime}}{\left[\bar{\omega}_{k}{ }^{2}+\varepsilon_{k}{ }^{2}\left(\omega_{T}\right)\right]-\omega_{T}^{2}} \overline{\mathbf{s}}_{k} \exp \left[i \omega_{T} t\right]$,

where we define $\varepsilon_{k}^{2}$ as

$\varepsilon_{k}^{2}\left(\omega_{T}\right) \equiv\left\langle\overline{\mathbf{s}}_{k}, \delta \mathcal{H}\left(\omega_{T}\right) \overline{\mathbf{s}}_{k}\right\rangle$.

The term $\varepsilon_{k}^{2}$ represents a complex perturbation to $\bar{\omega}_{k}^{2}$. 


\subsection{Love numbers}

For planetary-scale problems we may introduce tidal Love numbers (Love 1911; Shida 1912; Farrell 1972), which provide a mapping between the forcing potential and response. This mapping is expressed in the spherical harmonic domain as

$\mathbf{s}(\mathbf{r} ; t)=\sum_{\ell} \frac{h_{\ell}(r)}{g}\left[\hat{\mathbf{r}} \cdot \nabla \Psi_{\ell m}(\mathbf{r} ; t)\right]+\sum_{\ell} \frac{l_{\ell}(r)}{g}\left[\nabla_{1} \Psi_{\ell m}(\mathbf{r} ; t)\right]$,

where $h$ is the Love number associated with radial displacement, $s_{r}$, and $l$ is the Love number associated with tangential displacements, $s_{\theta}$ and $s_{\psi}$. Given the linear nature of this system we may derive expressions for $h$ and $l$ using results from Section 2.2. In the case of the ENM method, simple expressions for these parameters are not possible since we do not know the form of $\mathbf{B}$. In contrast, in the case of the DS method, combining eqs (30), (37) and (39) yields

$h_{\ell}\left(a ; \omega_{T}\right)=\frac{1}{g} \sum_{n} \frac{{ }_{n} \bar{U}_{\ell}(a)}{\left[\bar{\omega}_{n}^{2}+\varepsilon_{n}{ }^{2}\left(\omega_{T}\right)\right]-\omega_{T}^{2}} \int_{0}^{a} \rho(r) \frac{r^{\ell+1}}{a^{\ell}}\left[\ell_{n} \bar{U}_{\ell}(r)+\sqrt{\ell(\ell+1)}{ }_{n} \bar{V}_{\ell}(r)\right] \mathrm{d} r$

and

$l_{\ell}\left(a ; \omega_{T}\right)=\frac{1}{g \sqrt{\ell(\ell+1)}} \sum_{n} \frac{{ }_{n} \bar{V}_{\ell}(a)}{\left[\bar{\omega}_{n}{ }^{2}+\varepsilon_{n}{ }^{2}\left(\omega_{T}\right)\right]-\omega_{T}{ }^{2}} \int_{0}^{a} \rho(r) \frac{r^{\ell+1}}{a^{\ell}}\left[\ell_{n} \bar{U}_{\ell}(r)+\sqrt{\ell(\ell+1)}{ }_{n} \bar{V}_{\ell}(r)\right] \mathrm{d} r$,

where we have evaluated the Love numbers at Earth's surface, $r=a$ (this will be implicitly assumed hereafter), and we have made explicit the Love number dependence on the frequency of the forcing, $\omega_{T}$. As discussed above, the spherical-symmetry in the adopted Earth model results in a $(2 \ell+1)$-degeneracy in the eigenmodes and thus the expressions (40-41) exhibit no $m$ dependence. However, the tidal forcing does include such dependence, and this is reflected in eq. (39). The anelastic character is captured in $\varepsilon^{2}$ which gives rise to complex $h\left(\omega_{T}\right)$ and $l\left(\omega_{T}\right)$ values. $\operatorname{Re}\left\{h\left(\omega_{T}\right)\right\}$ represents the in-phase response and $\operatorname{Im}\left\{h\left(\omega_{T}\right)\right\}$ represents the out-of-phase response, relative to the forcing potential. The same is true for $l\left(\omega_{T}\right)$. Wahr (1981a) showed that the Love numbers may be found by the appropriate superposition of the normal modes. However, in treating anelasticity, Wahr \& Bergen (1986) introduced approximations that yielded expressions for the complex Love numbers that do not match those in eqs (40-41).

\subsection{The traditional normal mode (TNM) theory}

The most widely used theory to predict the effects of anelasticity on the body tide was formulated by Wahr \& Bergen (1986), the last of a series of canonical studies covering the effects on body tides of rotation, free-core nutation and anelasticity (Wahr 1981a,b; Wahr \& Bergen 1986, respectively). We will hereafter refer to their approach as the 'traditional normal mode' (TNM) theory. The TNM theory assumes that anelasticity perturbs existing elastic modes but would not introduce new modes, so that

$\bar{\omega}_{k} \rightarrow \bar{\omega}_{k}+\delta \hat{\omega}_{k}$,

where $\delta \hat{\omega}_{k}$ is a real number. This perturbation, $\delta \hat{\omega}$, is found by solving the following system:

$\operatorname{Re}\left\{\mathcal{H}\left(\omega_{T}\right)\right\} \hat{\mathbf{s}}_{k}-\hat{\omega}_{k}^{2} \hat{\mathbf{s}}_{k}=\mathbf{0}$,

where $\mathcal{H}\left(\omega_{T}\right)$ is fixed to the forcing frequency of interest, $\omega_{T}$, and hence $\hat{\omega}_{k}=\bar{\omega}_{k}+\delta \hat{\omega}_{k}$. In this case, $\mathcal{H}$ is no longer a function of frequency and $\left[\hat{\omega}_{k}{ }^{2}, \hat{\mathbf{s}}_{k}\right]$ are interpreted as eigenfrequency-eigenfunction pairs.

Strictly speaking, $\hat{\mathbf{s}}_{k}$ and $\hat{\omega}_{k}$ are not eigenmodes of a frequency-dependent Earth. True eigenmodes are computed by solving eq. (16); that is, eigenmodes 'see' the Earth at their eigenfrequency, $\mathcal{H}=\mathcal{H}\left(\omega_{k}\right)$. Moreover, eq. (16) involves $\mathcal{H}$, not just its real part, and thus admits the possibility of relaxation behaviour (and relaxation modes). As discussed above, these relaxation modes are essential to accurately capture anelastic behaviour at frequencies beyond those of the dynamic modes (Fig. 1a; or see Lau et al. 2015). Henceforth, we will use the symbol $\hat{X}$ to denote any variable $X$ associated with the pseudo-eigenvalue problem (eq. 43).

Fig. 1(b) is a schematic illustration of the variation in the real part of modulus $M$ across a large frequency band. We show two hypothetical models for $\operatorname{Re}\{M\}:$ A and B. If we were to solve the full eigenvalue problem as formulated within the seismological community we would omit the relaxation modes and include only dynamic modes. With this subset of modes, the application of a tidal force at $\omega_{T}$ (where it is assumed that $\omega_{T}$ lies far away from the seismic band) would lead to the same tidal predictions for both models A and B. The lowest frequency sampled by dynamic modes of a typical Earth model (e.g. PREM, Dziewonski \& Anderson 1981) is approximately $1 \mathrm{hr}^{-1}$. Thus, behaviour at lower frequencies than this, where A and B begin to diverge, is not captured. In contrast, applying Lau et al.'s (2015) ENM theory which includes relaxation modes, or the DS variant, would yield distinct tidal predictions for models A and B. This is illustrated by the sampling of relaxation modes at different frequencies for the different models (Fig. 1b).

Finally, in the TNM method, which solves the pseudo-eigenvalue problem (eq. 43), the resulting pseudo-eigenmodes are limited to (an approximated version of the dynamic modes. Specifically, the TNM treatment softens $\operatorname{Re}\{M\}$ to the values relevant for the Earth at $\omega_{T}$ and this is the Earth that the pseudo-eigenmodes sample, irrespective of their eigenfrequency value. This is shown schematically by the green dashed lines in Fig. 1(b). 
We have not yet discussed how a fully complex response is calculated using the TNM theory. Wahr \& Bergen (1986) solved eq. (43), which is analogous to solving an elastic problem with elastic moduli fixed to a reduced value of $\boldsymbol{\Lambda}\left(\omega_{T}\right)$, and this yielded real $\left[\hat{\omega}_{k}{ }^{2}, \hat{\mathbf{s}}_{k}\right]$. These eigenmodes were then combined to compute $\operatorname{Re}\{\hat{h}\}$. (This approach is in contrast to the normal mode treatment of Tromp \& Dahlen (1990) and Lognonné (1991) who computed complex eigenmodes.) Next, assuming that the effects of anelasticity are small enough that perturbations in $\boldsymbol{\Lambda}(\omega)$ scaled linearly to perturbations in $h$, they computed the imaginary component of $\hat{h}$ using the following expression

$\operatorname{Im}\{\hat{h}(\omega)\}=\int_{\mathrm{CMB}}^{a} \frac{\partial \operatorname{Re}\{\hat{h}(\omega)\}}{\partial M(\omega, r)} \operatorname{Im}\{\delta M(\omega, r)\} \mathrm{d} r$.

Wahr \& Bergen (1986) estimated the partial derivative in this expression as

$\frac{\partial \operatorname{Re}\{\hat{h}(\omega)\}}{\partial M(\omega, r)} \approx \frac{\operatorname{Re}\{\hat{h}(\omega)\}-\bar{h}}{\operatorname{Re}\{M(\omega)\}-\bar{M}}$,

where $\bar{h}$ may be found by setting $\varepsilon^{2} \rightarrow 0$ in eq. (40). They discuss in detail choices of $\omega$ that yield stable estimates of the partial derivative.

\section{COMPARISON OF PREDICTIONS USING THE TNM AND DS THEORIES}

Predictions of body tides have numerous applications, including: correcting a wide range of space-geodetic observables for the body tide signal (e.g. IERS standards, Cartwright \& Petit 2004; Petit \& Luzum 2010); inferring anelastic structure from observations of tidal phase lags (e.g. Ray et al. 2001; Benjamin et al. 2006); and, potentially, estimating long-wavelength elastic and density variations in the mantle using tidal tomography (Métivier \& Conrad 2008; Latychev et al. 2009; Qin et al. 2014; Lau et al. 2015). In this regard, given the same input anelastic structure (defined by the tensor $\boldsymbol{\Lambda}(\omega)$ ), the TNM and more accurate ENM/DS treatments of the body tide response of the Earth will predict different values of the frequency-dependent Love number $h(\omega)$. In this section, we present several simple numerical examples that highlight these differences (Section 3.1) and discuss their implications for the interpretation of tidal phase lags (Section 3.2).

\subsection{Numerical predictions}

In the results below, we adopt several models for the form of $\boldsymbol{\Lambda}(\omega)$ and predict the Love number $h$ as well as a phase parameter $\Upsilon$, defined as:

$\Upsilon \equiv \arctan \left(\frac{\operatorname{Im}\{h\}}{\operatorname{Re}\{h\}}\right)$.

To begin, we repeat the calculations based on the TNM treatment to demonstrate that we can reproduce the Wahr \& Bergen (1986) results for the M2 semi-diurnal body tide (see Fig. 2). These calculations incorporate the isotropic elastic and density structure of seismic model 1066A (Gilbert \& Dziewonski 1975) and perturb this structure by assuming a frequency-dependent $Q$ of the form:

$Q(\omega)=Q\left(\omega_{0}\right)\left(\frac{\omega}{\omega_{0}}\right)^{\alpha}$,

where $Q\left(\omega_{0}\right)$ is the attenuation at reference frequency $\omega_{0}$. We note that values for $\alpha$ across the seismic-tidal band have been shown to lie between 0.1 and 0.35 (Faul \& Jackson 2015) but as $\omega \rightarrow 0, \alpha \rightarrow 1$ if one adopts a Maxwell rheological model. The specific choice of phenomenological model is, however, not a focus of this paper. Wahr \& Bergen (1986) assumed dissipation in the shear modulus only, and they adopted the expression

$\delta \mu=\mu\left(\omega_{0}\right)\left\{\cot \left(\frac{\alpha \pi}{2}\right)\left[1-\left(\frac{\omega_{0}}{\omega}\right)^{\alpha}\right]+i\left(\frac{\omega_{0}}{\omega}\right)^{\alpha}\right\} \frac{1}{Q_{\mu}\left(\omega_{0}\right)}$.

In this equation, $Q_{\mu}$ is the attenuation associated with $\mu$. In tidal applications, it is common to exclude the contribution from bulk dissipation (e.g. Wahr \& Bergen 1986; Benjamin et al. 2006) since many $Q$ models derived from seismic observations (e.g. Dziewonski \& Anderson 1981; Durek \& Ekstrom 1996) exhibit very high values for $Q_{\kappa}$. More recent work, however, suggests that at these frequencies bulk dissipation may play an important role when considering porous media in the presence of partial melt (Takei \& Holtzman 2009). To incorporate bulk dissipation in our theory, the appropriate expression for $\kappa(\omega)$ need only be inserted in the relevant elements of the stress tensor $\boldsymbol{\Lambda}$ (see eq. 6). They furthermore adopted the depth-dependent attenuation models 'QMU' (Sailor \& Dziewonski 1978) and 'Model B' (Sipkin \& Jordan 1979). We performed predictions for three cases: (i) QMU with $\alpha=0.0$; (ii) QMU with $\alpha=0.15$ and (iii) Model B with $\alpha=0.09$. We note that as $\alpha \rightarrow 0$,

$\delta \mu \rightarrow \mu\left(\omega_{0}\right)\left[\frac{2}{\pi} \ln \left(\frac{\omega}{\omega_{0}}\right)+i\right] \frac{1}{Q_{\mu}\left(\omega_{0}\right)}$

(Kanamori \& Anderson 1977). The results for all three cases in Fig. 2 show excellent agreement between predictions of the complex Love number perturbation reported by Wahr \& Bergen (1986) and our predictions based on their approach.

We next compare predictions based on the TNM theory and the DS theory using two models of mantle anelasticity described by Benjamin et al. (2006). Both these models adopt the isotropic version of PREM (Dziewonski \& Anderson 1981) and the depth-dependent $Q$ structure 

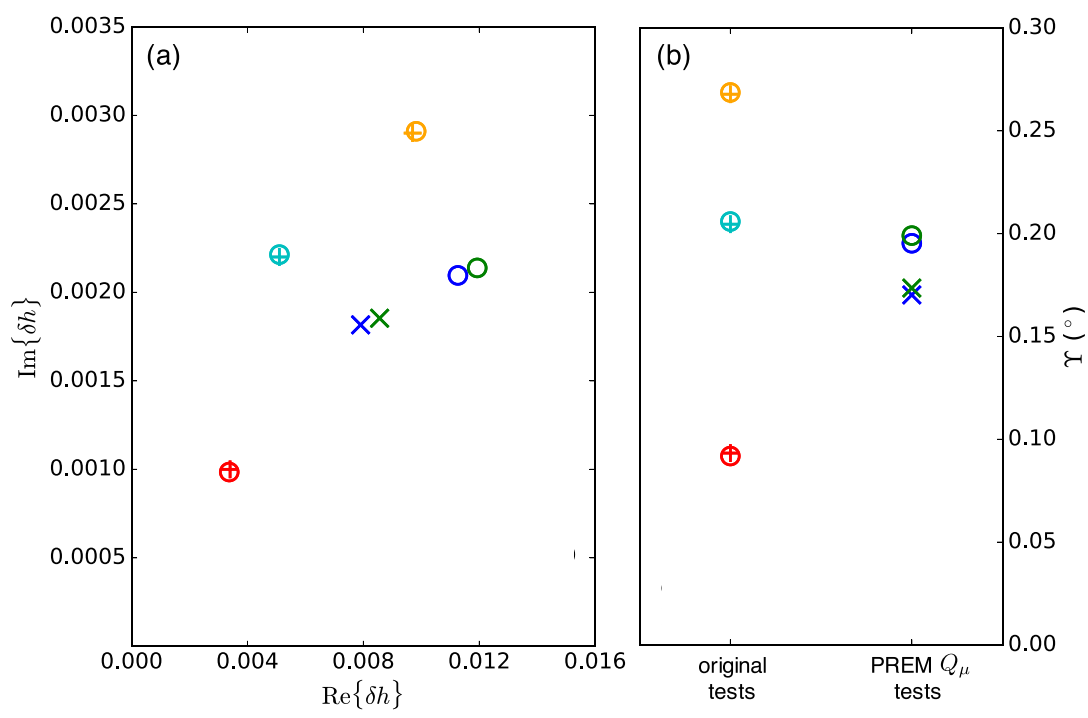

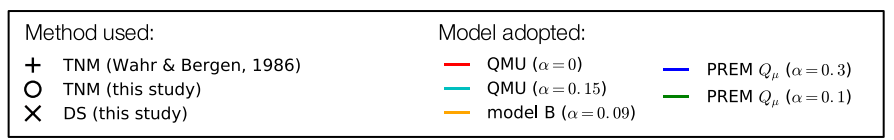

Figure 2. Predictions of (a) the perturbations to the degree-2 tidal Love number, $\operatorname{Re}\{\delta h\}$ and $\operatorname{Im}\{\delta h\}$, and (b) the predicted tidal phase lag, $\Upsilon$, calculated using the (circles) TNM and ( $\times$ s) DS (Lau et al. 2015) theories for five different Earth models distinguished on the basis of elastic and anelastic properties. The crosses $(+)$ are three results taken directly from the Wahr \& Bergen (1986) study that derived the TNM approach. The overlap of the crosses and circles demonstrates that we are able to correctly implement the TNM approach. We note that the reference frequencies, $\omega_{m}$, for the PREM $Q_{\mu}(\alpha=0.3)$ and PREM $Q_{\mu}(\alpha=0.1)$ Earth models are $3.09 \times 10^{-4} \mathrm{~Hz}$ and $5.0 \times 10^{-4} \mathrm{~Hz}$, respectively.

of $\operatorname{PREM}\left(Q_{\mu}\right.$ only) at the reference frequency, $f_{0}$, of $1 \mathrm{~Hz}$ (note we will use $f$ when referring to numerical values for frequencies where $f=$ $\omega / 2 \pi)$. The models also assume the frequency-dependent form of $Q$ given by eq. (47). The perturbation to $\mu$ in both cases is given by

$\delta \mu(\omega)=\left\{\begin{array}{l}\mu\left(\omega_{0}\right)\left[\frac{2}{\pi} \ln \left(\frac{\omega}{\omega_{0}}\right)+i\right] Q\left(\omega_{0}\right)^{-1}, \text { for } \omega \geq \omega_{m} \\ \mu\left(\omega_{0}\right)\left[\frac{2}{\pi}\left\{\ln \left(\frac{\omega_{m}}{\omega_{0}}\right)+\frac{1}{\alpha}\left[1-\left(\frac{\omega_{m}}{\omega}\right)^{\alpha}\right]\right\}+i\left(\frac{\omega_{m}}{\omega}\right)^{\alpha}\right] Q\left(\omega_{0}\right)^{-1}, \text { for } \omega<\omega_{m},\end{array}\right.$

where the free parameter $\omega_{m}$ is the frequency that defines the onset of frequency-dependent behaviour in $Q$. We adopt two choices for this frequency, $f_{m}$, (chosen from a subset of values shown in figs 6 and 7 of Benjamin et al. 2006): (i) $3.09 \times 10^{-4} \mathrm{~Hz}$, with $\alpha=0.2$; and (ii) 5.0 $\times 10^{-3} \mathrm{~Hz}$, with $\alpha=0.1$. Both these frequencies are significantly higher than the semi-diurnal frequency.

Fig. 2(a) shows that there is a systematic difference in the predictions based on the two theories. In both cases, the formulation of Wahr \& Bergen (1986) overestimates $\operatorname{Re}\{\delta h\}$ and $\operatorname{Im}\{\delta h\}$ by $\sim 50$ per cent and $\sim 15$ per cent, respectively. As a result, the traditional theory overestimates the lag angle $\Upsilon$ by $\sim 20$ per cent (Fig. $2 b$ ).

\subsection{Is intrinsic $Q$ the same as 'observed $Q$ '?}

In this section, we highlight the implications of our revision to the body tide theory for the interpretation of tidal observations. To begin, we define $\Upsilon$ as the observed phase lag at the planetary-scale with respect to the forcing and $\varphi$ as the phase lag between stress and strain for a material sample:

$\check{Q}^{-1} \approx \tan [\Upsilon(\omega)]=\frac{\operatorname{Im}\{h(\omega)\}}{\operatorname{Re}\{h(\omega)\}}$,

$Q^{-1} \approx \tan [\varphi(\omega)]=\frac{\operatorname{Im}\{Q(\omega)\}}{\operatorname{Re}\{Q(\omega)\}}$

We refer to the quantity $\breve{Q}^{-1}$ as the apparent attenuation (i.e. the attenuation manifest in observations and to which tidal calculations discussed above are compared) and $Q^{-1}$ as the intrinsic (i.e. material) attenuation. It is common to assume that $\check{Q}^{-1}$ and $Q^{-1}$ are equivalent (Ray et al. 2001; Benjamin et al. 2006). However, while the difference between the two parameters is subtle, we demonstrate in this section that distinguishing between them is crucial for a robust understanding of Earth's anelastic response across a wide frequency band.

To illustrate the relevant arguments, consider Fig. 3, which shows a schematic system involving a 1-D analogue of anelastic behaviour. The spring-dashpot component is left incomplete to imply any linear combination of mechanical elements may be applied in this scenario to represent the transient component (e.g. an absorption band, Kanamori \& Anderson 1977). In addition to this, several components are 


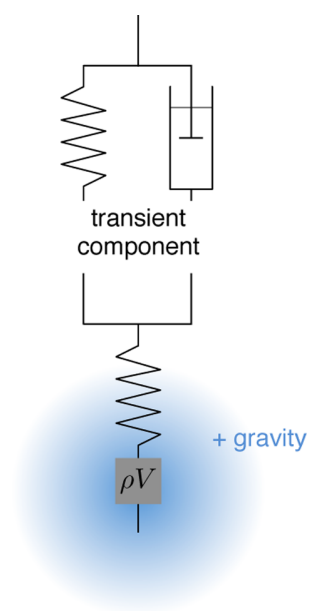

Figure 3. A schematic illustration of the system described by eq. (16). The Kelvin-Voigt element is left incomplete to imply any combination of spring-dashpot elements to form a transient component to the system. Additional components to the system include the mass component, $\rho V$, to represent the presence of inertia and the blue shading to represent the presence of self-gravity (see the text).

included in Fig. 3 which illustrate processes ongoing across the entire planet: inertia and self-gravity. The former is represented by the mass component, $\rho V$, and the latter by the blue shading. In previous studies, planetary-scale phase lags, $\Upsilon$, have been interpreted as reflecting only the intrinsic anelastic property of the material that forms the building blocks of the planet (i.e. these studies have ignored the impact of the mass component and gravitational body force on the observed lag; e.g. Wahr \& Bergen 1986; Ray et al. 2001; Benjamin et al. 2006). However, consideration of all these issues is required to accurately interpret the anelastic response of the Earth. In the following, we will use numerical calculations to consider, in turn, each factor that distinguishes these two interpretations (inertia, self-gravity, energy considerations, as defined below) and demonstrate how the intrinsic attenuation, $Q^{-1}$, may be extracted from the apparent attenuation, $\check{Q}^{-1}$.

We note that different processes (e.g. body waves or tides) will be sensitive to different regions of the spatially varying $Q^{-1}$ structure of the Earth (see e.g. the $Q$ model of Durek \& Ekstrom 1996). This issue will, in general, complicate the mapping between $Q$ and $\check{Q}$ and we address it in detail in future work. In the discussion below, we avoid this complication by adopting a simple anelastic Earth model and focusing on observables with comparable spatial sensitivity. Nevertheless, the calculations are sufficiently complex to demonstrate the main points in our present argument.

The calculations discussed below adopt the elastic and density structure of the seismic model PREM (Dziewonski \& Anderson 1981). We will assume a spatially uniform $Q_{\mu}$ value of 250 throughout the mantle, and frequency dependence governed by eq. (50) with parameter values $\alpha=0.3, f_{0}=1 \mathrm{~Hz}$, and $f_{m}=3.09 \times 10^{-4} \mathrm{~Hz}$. Fig. 4 (black line) shows the resulting frequency dependence of the intrinsic attenuation $Q^{-1}$.

The orange line on the same figure is the prediction of $\check{Q}^{-1}$ based on the theory of Wahr \& Bergen (1986) applied to the above model of anelastic Earth structure. This prediction clearly differs from the imposed $Q^{-1}$. If, for example, one were to assume that this prediction was equivalent to intrinsic attenuation $Q^{-1}$ (e.g. Ray et al. 2001; Benjamin et al. 2006), then one would overestimate the latter by a factor of $\sim 2$ within the semi-diurnal frequency.

It is particularly important to emphasize the implications of the above error for inferences of the frequency dependence of $Q^{-1}$. Tidal and seismic observations sample anelastic behaviour at a relatively small set of frequencies. For example, let us presume that the red crosses labelled A and B in Fig. 4 represent observations of attenuation with two distinct frequencies: a semi-diurnal tide, with frequency of $12 \mathrm{hr}^{-1}$, and the seismic free oscillation ${ }_{0} S_{2}$, with frequency of $\sim 1 \mathrm{hr}^{-1}$. (Lau et al. (2015) demonstrated that ${ }_{0} S_{2}$ deformation is responsible for 95 per cent of the semi-diurnal body tide response, and thus both observations sample very similar $Q$ structure (see Anderson \& Minster 1979; Benjamin et al. 2006).) The goal in the application of TNM theory of body tides would be to accurately predict the observation A, and this might lead, for example, to the orange line in Fig. 4. As noted above, whilst this is in fact a prediction of apparent attenuation, it has been commonly interpreted as an accurate proxy for the intrinsic attenuation at this frequency. In contrast, seismic normal mode measurements of the decay time of the ${ }_{0} S_{2}$ mode would infer the correct value of intrinsic attenuation $Q(\omega)^{-1}$ at $1 \mathrm{hr}^{-1}$, that is, the value at point B. Using these two observables, one might suppose a variety of trends in the intrinsic attenuation between $\mathrm{A}$ and $\mathrm{B}$, but a simple straight line (or equivalently, a constant exponent in $\omega^{\alpha}$ ) would clearly yield an inaccurate model for the true trend.

The solid cyan line in Fig. 4 shows the trend in $\breve{Q}^{-1}$ computed using the DS theory. Of course, it would also be incorrect to assume that these predictions reflect the intrinsic attenuation, but the following question arises: If calculations of $\breve{Q}^{-1}$ in the tidal band based on the DS (or ENM) theory and a specific model of anelastic structure, fit available observations, can one extract a robust estimate of intrinsic attenuation and (through a comparison with seismic observation of attenuation) its frequency dependence? To answer this question requires that we consider and correct for each of the physical cases that differentiate the intrinsic attenuation from the observed (and modelled) response of the Earth (i.e. the apparent attenuation). In this regard, in the next three sections we outline a set of progressive corrections that accurately map $\check{Q}^{-1}(\omega)$, as computed using the DS and ENM methods, into $Q^{-1}(\omega)$. 


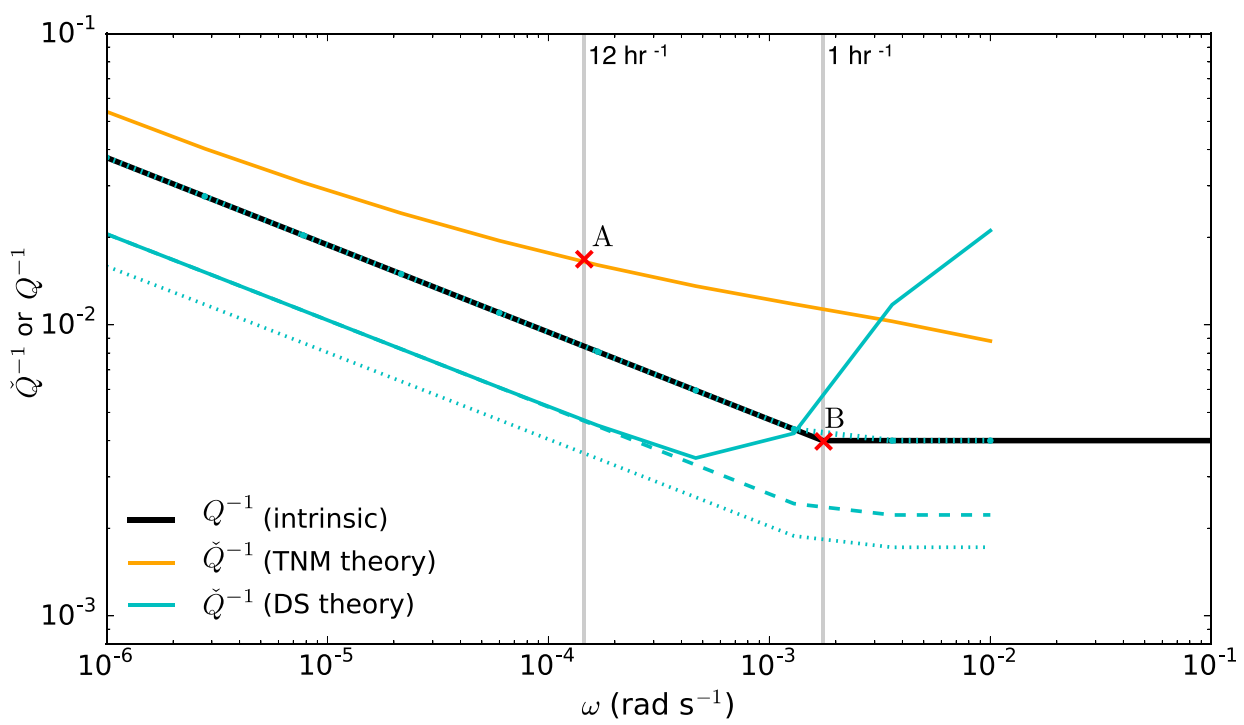

Figure 4. Intrinsic attenuation, $Q^{-1}$ (black line), as a function of frequency associated with the anelastic Earth model described in the text (see Section 3.2). The remaining lines are various predictions of apparent attenuation, $\breve{Q}^{-1}$, based on the same Earth model: orange line-prediction using the TNM theory; solid cyan line - prediction using the DS theory; dashed cyan line — correction to the solid cyan line to remove the impact of inertia; dotted cyan line-correction to the solid cyan line to remove the impact of both inertia and self-gravity; dashed-dotted cyan line (coincident with the black line) - calculation in which the solid cyan line is mapped into intrinsic attenuation using the expression (61). The two highest frequency predictions using the DS theory (solid cyan line) have been multiplied by -1 so that they may be plotted on the same log-log scale. (We note in this regard that a system will oscillate in antiphase with a forcing if the forcing has a frequency well above the resonant frequency of the system.) Points A and B mark frequencies that can be realistically observed on Earth: the semi-diurnal tide M2 and the ${ }_{0} S_{2}$ normal mode, respectively. See Section 3.2 for further discussion.

\subsection{Inertia}

Consider, once again, the apparent attenuation in Fig. 4 computed using the DS theory. At frequencies lower than $\sim 4 \times 10^{-4} \mathrm{rad} \mathrm{s}^{-1}$ the slope of $\check{Q}^{-1}(\omega)$ accurately tracks the trend in intrinsic attenuation $Q^{-1}$, however the values on the former are offset to lower values than those on the latter. The difference between the predictions of $\check{Q}^{-1}$ based on the TNM and DS (or ENM) theories is to be expected given the results in Fig. 2(b). The large swing in $\check{Q}^{-1}$ predicted by the DS theory at frequencies above $\sim 4 \times 10^{-4} \mathrm{rad} \mathrm{s}^{-1}$ is due to resonant effects associated with the dynamic eigenmodes (the lowest frequency of which is $\approx 1 \mathrm{hr}^{-1}$ ).

The first correction toward mapping the $\check{Q}^{-1}$ prediction computed using the DS (or ENM) method into an estimate of the intrinsic attenuation thus involves the removal of this resonance effect. This is equivalent to neglecting inertia in the calculation by invoking the limit $\omega_{T} \rightarrow 0$ in eqs (40) and (41). The result of applying this correction is shown by the dashed cyan line in Fig. 4, which now accurately follows (with a constant offset) the frequency dependence of $Q^{-1}$.

\subsection{Self-gravity}

The tidal force causes expansional deformation on the Earth and acting against this deformation are the elastic restoring forces and inward self-gravity which are embedded in the operator $\mathcal{H}$ (eq. 11). In the case of the body tide problem, both forces in $\mathcal{H}$ resist the expansion and as such, the self-gravity term provides an additional effective rigidity to the elastic rigidity. We note that Efroimsky (2012a, 2013) discusses this physics in relation to Earth and super-Earths and argues that the effect cannot be ignored when considering tidal dissipation on such planets. The same process acts on Earth, and contributes to a difference in intrinsic and apparent attenuation. To remove gravity from the general equations, we take the limits $\Phi \rightarrow 0$ and $\phi \rightarrow 0$, so that the operator $\mathcal{H}$ in eq. (11) becomes

$\rho \mathcal{H} \mathbf{s}=-\nabla \cdot(\boldsymbol{\Lambda}: \nabla \mathbf{s})$.

In this case, the only restoring force arises from the elastic properties of the Earth. In the schematic Fig. 3, this would be equivalent to removing the body force from the system. This procedure represents the second correction necessary to map $\check{Q}^{-1}$ to $Q^{-1}$, and applying it to the dashed cyan line in Fig. 4 yields the result shown by the dotted cyan line. The resultant reduction in the attenuation is due to the increase of $\operatorname{Re}\{h\}$ associated with a lowering of the effective rigidity when gravitational body forces are removed. Moreover, the constant shift downwards from dashed to dotted cyan line reflects the fact that the contribution from gravity is independent of frequency. We further note that self-gravity would have the opposite effect if the induced deformation was compressional, acting in the same direction as the external forcing, thus lessening the effective rigidity. 


\subsection{Energy considerations}

In this section we revisit eq. (40), though we note that all the results below hold for eq. (41). Removing the impact of inertia from eq. (40), as discussed in Section 3.3, yields

$h_{\ell}\left(\omega_{T}\right)=\frac{1}{g} \sum_{n} \frac{{ }_{n} \bar{U}_{\ell}}{\bar{\omega}_{n}{ }^{2}+\varepsilon_{n}{ }^{2}} \int_{0}^{a} \rho(r) \frac{r^{\ell+1}}{a^{\ell}}\left[\ell_{n} \bar{U}_{\ell}(r)+\sqrt{\ell(\ell+1)_{n}} \bar{V}_{\ell}(r)\right] \mathrm{d} r$.

Following Section 3.4, we note that the eigenfunctions and eigenfrequencies in these expressions are associated with a system without self-gravity (the operator $\mathcal{H}$ is given by eq. 53).

To complete the mapping between $\breve{Q}^{-1}$ and $Q^{-1}$ we recall arguments made by O'Connell \& Budiansky (1978) who, in a similar vein as the present study, set out to clarify the distinction between the intrinsic $Q^{-1}$ of a material and measures of $\check{Q}^{-1}$ in several phenomenological processes (e.g. travelling plane waves, standing waves). In particular, we interpret $Q^{-1}$ using energy considerations as briefly mentioned in Section 2.1. To begin, we rewrite the definition in eq. (9) as

$Q^{-1} \equiv \frac{\Delta W}{W}$

where $\Delta W$ and $W$ represent the dissipated and stored energies per cycle, respectively. When considering a vibrating system close to resonance, one may treat the system as having only one degree of freedom; we can thus identify the pair $\Delta W_{k}$ and $W_{k}$ for each eigenmode, $k$. We note that such an approach already exists in the seismic normal mode literature (see Dahlen \& Tromp 1998). Accordingly, for the particular case described by eq. (54), invoking the definition given by eq. (51) yields (see Appendix B):

$$
\begin{aligned}
\check{Q}^{-1} & =\frac{\sum_{k} \chi_{k} \operatorname{Im}\left\{\varepsilon_{k}{ }^{2}\right\}}{\sum_{k} \chi_{k}\left(\bar{\omega}_{k}^{2}+\operatorname{Re}\left\{\varepsilon_{k}{ }^{2}\right\}\right)} \\
& =\frac{\sum_{k} \chi_{k} \int_{V} \nabla \mathbf{s}_{k}^{*}: \operatorname{Im}\left\{\left(\boldsymbol{\Lambda}\left(\omega_{T}\right)\right\}: \nabla \mathbf{s}_{k} \mathrm{~d} V\right.}{\sum_{k} \chi_{k}\left(\bar{\omega}_{k}^{2} \int_{V} \rho \mathbf{s}_{k}{ }^{*} \cdot \mathbf{s}_{k} \mathrm{~d} V+\int_{V} \nabla \mathbf{s}_{k}^{*}: \operatorname{Re}\left\{\delta \boldsymbol{\Lambda}\left(\omega_{T}\right)\right\}: \nabla \mathbf{s}_{k} \mathrm{~d} V\right)},
\end{aligned}
$$

where

$\chi_{k}=\frac{\int_{0}^{a} \rho(r) \frac{r^{\ell+1}}{a^{\ell}}\left[\ell_{n} \bar{U}_{\ell}(r)+\sqrt{\ell(\ell+1)} \bar{V}_{\ell}(r)\right] \mathrm{d} r}{\bar{\omega}_{k}^{4}+\operatorname{Im}\left\{\varepsilon_{k}^{2}\right\}^{2}}$.

$\chi_{k}$ represents a weighting of each mode which reflects excitation by the tidal forcing.

The first term in the denominator of eq. (56) is the kinetic energy (this term is equivalent to the stored elastic energy) and the second term is the energy correction associated with dispersion. The numerator is the energy loss due to dissipation. That is,

$\Delta W_{k}=\chi_{k} \int_{V} \nabla \mathbf{s}_{k}^{*}: \operatorname{Im}\left\{\boldsymbol{\Lambda}\left(\omega_{T}\right)\right\}: \nabla \mathbf{s}_{k} \mathrm{~d} V$

where, for the specific $Q$ model we have prescribed (i.e. where all dissipation occurs in shear energy)

$\Delta W_{k}=\chi_{k} \int_{V} 2 \mu\left(\mathbf{d}_{k}^{*}: \mathbf{d}_{k}\right) Q_{\mu}(\omega)^{-1} \mathrm{~d} V$

In this expression $\mathbf{d}$ is the deviatoric strain. Since the energy associated with dispersion is much smaller than the elastic energy, one can make the following approximation

$W_{k} \approx \chi_{k} \bar{\omega}_{k}^{2} \int_{V} \rho \mathbf{s}_{k}^{*} \cdot \mathbf{s}_{k} \mathrm{~d} V$

where, if the system included self-gravity, an additional gravitational potential energy term would contribute to $W$. Our final, complete form for the mapping between $\breve{Q}^{-1}$ and $Q^{-1}$ is thus given by

$\check{Q}^{-1}\left(\omega_{T}\right)=\left(\frac{\sum_{k} \chi_{k} \int_{V} 2 \mu\left(\mathbf{d}_{k}^{*}: \mathbf{d}_{k}\right) \mathrm{d} V}{\sum_{k} \chi_{k} \bar{\omega}_{k}^{2} \int_{V} \rho \mathbf{s}_{k}^{*} \cdot \mathbf{s}_{k} \mathrm{~d} V}\right) Q^{-1}\left(\omega_{T}\right)$.

This expression relates the apparent attenuation arising from a planetary-scale phase lag in the tidal response to the intrinsic attenuation of the material that comprises the planet. The simple mapping between $\breve{Q}^{-1}$ and $Q^{-1}$ is only possible in our specialized case where $Q^{-1}$ is spatially uniform. Using this expression in Fig. 4 yields a line that precisely matches the intrinsic attenuation $Q(\omega)^{-1}$ adopted in the simulations.

We note that Nowick \& Berry (1972, Appendix A) present an analysis of the energy budget for the case of a 1-D spring-dashpot system. The mapping between $\breve{Q}^{-1}$ and $Q^{-1}$ for their toy example is analogous to our eq. (61).

\section{CONCLUDING REMARKS}

Lau et al. (2015) presented an ENM theory for predicting body tides on an aspherical, rotating and anelastic Earth. In this paper, we have elaborated on the ENM theory, as well as a relatively minor variant, the DS approach, and have explored the implications of the theory for 
the modelling of anelastic effects and interpretations of relevant observations. Our theory differs from the TNM theory of Wahr \& Bergen (1986) in several significant ways:

(i) it is based on a pure normal mode formalism that is in accord with, and extends, the theory used in seismic free oscillation research by incorporating a set of relaxation modes that are not considered in the TNM theory; and

(ii) it treats anelastic effects on each mode by determining the imaginary perturbation to their eigenfrequencies. The TNM theory, in contrast, estimates the imaginary part of the full response by considering the imaginary part of the modulus perturbation.

Given the same model for anelastic Earth structure, the more accurate ENM (or DS) theory will predict a different perturbations to tidal Love numbers than the TNM method and this will have implications not only for the inferences of the anelastic structure of the Earth but also for model-based corrections to geodetic data that are applied to remove the body tide signal.

We have also demonstrated that previous analyses of the tidal phase lag observation have, in effect, assumed that the tidal lag angle on a planetary-scale is the same as the loss angle (i.e. the lag angle between the stress and strain acting on a material sample, see eq. 52). We have shown that if inertia, self-gravity and energy considerations are taken into account, the true loss angle, or equivalently the intrinsic $Q^{-1}$, may be accurately extracted from the observed planetary phase lag, or apparent $\check{Q}^{-1}$. If these issues are not addressed, it is likely that systematic biases in the estimates of the frequency dependence of $Q^{-1}$ will arise. Our formulation provides a rigorous procedure for estimating intrinsic attenuation in the tidal band and for connecting measurements of dissipation across frequencies that span tidal to seismic processes.

As a final note, we emphasize that the generalized treatment of anelasticity described here and in Lau et al. (2015) may be applied to investigate the response of other planetary bodies to tidal forcing. Indeed, planetary science provides a far broader range of physical conditions than the geophysical applications we have focused on, and this range will no doubt sample regimes with sensitivities vastly different from those explored in our simple numerical examples. As one obvious example, the impact of self-gravitation on the anelastic response of massive super-Earths will be significantly larger than the already important effect revealed in Fig. 4. More generally, observational constraints on other terrestrial bodies are often limited to an estimate of the tidal Love number, and the correct interpretation of this number is then clearly critical to any assessment of physical conditions of that body.

\section{ACKNOWLEDGEMENTS}

We acknowledge several insightful comments by John Wahr, to whom we dedicate this manuscript. This work was supported by NSF EAR1464024, NSF EAR-1215061, and Harvard University. We additionally thank the reviewers Michael Efroimsky and Mark Panning for their constructive comments on the manuscript.

\section{REFER E N CES}

Al-Attar, D., 2007. A solution of the elastodynamic equation in an anelastic earth model, Geophys. J. Int., 171(2), 755-760.

Al-Attar, D., Woodhouse, J.H. \& Deuss, A., 2012. Calculation of normal mode spectra in laterally heterogeneous earth models using an iterative direct solution method, Geophys. J. Int., 189(2), 1038-1046.

Anderson, D.L. \& Minster, J.B., 1979. The frequency dependence of Q in the Earth and implications for mantle rheology and Chandler wobble, Geophys. J. Int., 58(2), 431-440.

Barnhoorn, A., Jackson, I., Fitz Gerald, J.D. \& Aizawa, Y., 2007. Suppression of elastically accommodated grain-boundary sliding in high-purity MgO, J. Eur. Ceram. Soc., 27(16), 4697-4703.

Bellis, C. \& Holtzman, B., 2014. Sensitivity of seismic measurements to frequency-dependent attenuation and upper mantle structure: An initial approach, J. geophys. Res., 119(7), 5497-5517.

Benjamin, D., Wahr, J., Ray, R.D., Egbert, G.D. \& Desai, S.D., 2006. Constraints on mantle anelasticity from geodetic observations, and implications for the J 2 anomaly, Geophys. J. Int., 165(1), 3-16.

Cartwright, D.E. \& Petit, J.-M., 2004. IERS Conventions (2003), International Earth Rotation and Reference Systems Service, IERS Technical Note No. 32.

Dahlen, F.A., 1968. The normal-modes of a rotating, elliptical Earth, Geophys. J. Int., 16(4), 329-367.

Dahlen, F.A. \& Tromp, J., 1998. Theoretical Global Seismology, Princeton University Press.

Day, W.A., 1971a. Time-reversal and the symmetry of the relaxation function of a linear viscoelastic material, Arch. Ration. Mech. Anal., 40(3), $155-159$.

Day, W.A., 1971b. Restrictions on relaxation functions in linear viscoelasticity, Q. J. Mech. Appl. Math., 24(4), 487-497.
Dehant, V., 1987. Tidal parameters for an inelastic earth, Phys. Earth planet. Inter., 49(1-2), 97-116.

Dehant, V., Defraigne, P. \& Wahr, J.M., 1999. Tides for a convective Earth, J. geophys. Res., 104(B1), 1035-1058.

Durek, J.J. \& Ekstrom, G., 1996. A radial model of anelasticity consistent with long-period surface-wave attenuation, Bull. seism. Soc. Am., 86(1A), 144-158.

Dziewonski, A.M. \& Anderson, D.L., 1981. Preliminary reference Earth model, Phys. Earth planet. Inter., 25(4), 297-356.

Edmonds, A.R., 1960. Angular Momentum in Quantum Mechanics, Princeton University Press.

Efroimsky, M., 2012a. Tidal dissipation compared to seismic dissipation: in small bodies, earths, and super-earths, Astrophys. J., 746(2), 150, doi:10.1088/0004-637X/746/2/150.

Efroimsky, M., 2012b. Bodily tides near spin-orbit resonances, Celest. Mech. Dyn. Astron., 112(3), 283-330.

Efroimsky, M., 2013. Erratum: tidal dissipation compared to seismic dissipation: in small bodies, earths, and super-earths (2012, ApJ, 746, 150), Astrophys. J., 763(2), 150, doi:10.1088/0004-637X/763/2/150.

Farrell, W.E., 1972. Deformation of the Earth by surface loads, Rev. Geophys., 10(3), 761.

Faul, U. \& Jackson, I., 2015. Transient creep and strain energy dissipation: an experimental perspective, Annu. Rev. Earth Planet. Sci., 43(1), 541-569.

Gilbert, F., 1971. Excitation of the normal modes of the Earth by earthquake sources, Geophys. J. Int., 22(2), 223-226.

Gilbert, F. \& Dziewonski, A.M., 1975. An application of normal mode theory to the retrieval of structural parameters and source mechanisms from seismic spectra, Phil. Trans. R. Soc. A, 278(1280), 187-269.

Gribb, T.T. \& Cooper, R.F., 1998. Low-frequency shear attenuation in polycrystalline olivine: grain boundary diffusion and the physical 
significance of the Andrade model for viscoelastic rheology, J. geophys. Res., 103(B11), 27 267-27 279.

Hara, T., Tsuboi, S. \& Geller, R.J., 1993. Inversion for laterally heterogeneous upper mantle S-wave velocity structure using iterative waveform inversion, Geophys. J. Int., 115(3), 667-698.

Jackson, I., 1993. Progress in the experimental study of seismic wave attenuation, Annu. Rev. Earth Planet. Sci., 21(1), 375-406.

Jackson, I. \& Faul, U.H., 2010. Grainsize-sensitive viscoelastic relaxation in olivine: towards a robust laboratory-based model for seismological application, Phys. Earth planet. Inter., 183(1-2), 151-163.

Jackson, I., Fitzgerald, J.D., Faul, U.H. \& Tan, B.H., 2002. Grain-sizesensitive seismic wave attenuation in polycrystalline olivine, J. geophys. Res., 107(B12), ECV 5-1-ECV 5-16.

Kanamori, H. \& Anderson, D.L., 1977. Importance of physical dispersion in surface wave and free oscillation problems: review, Rev. Geophys., 15(1), $105-112$.

Latychev, K., Mitrovica, J.X., Ishii, M., Chan, N.-H. \& Davis, J.L., 2009. Body tides on a 3-D elastic Earth: toward a tidal tomography, Earth planet. Sci. Lett., 277(1-2), 86-90.

Lau, H.C., Yang, H.-Y., Tromp, J., Mitrovica, J.X., Latychev, K. \& Al-Attar, D., 2015. A normal mode treatment of semi-diurnal body tides on an aspherical, rotating and anelastic Earth, Geophys. J. Int., 202(2), 13921406.

Lee, L.C., Morris, S.J.S. \& Wilkening, J., 2011. Stress concentrations, diffusionally accommodated grain boundary sliding and the viscoelasticity of polycrystals, Proc. R. Soc. A, 467(2130), 1624-1644.

Lekić, V., Matas, J., Panning, M. \& Romanowicz, B., 2009. Measurement and implications of frequency dependence of attenuation, Earth planet. Sci. Lett., 282(1-4), 285-293.

Lognonné, P., 1991. Normal modes and seismograms in an anelastic rotating Earth, J. geophys. Res., 96(B12), 20 309-20 319.

Love, A.E.H., 1911. Some Problems of Geodynamics, Cambridge Univ. Press.

McCarthy, C., Takei, Y. \& Hiraga, T., 2011. Experimental study of attenuation and dispersion over a broad frequency range: 2 . The universal scaling of polycrystalline materials, J. geophys. Res., 116(B9), B09207, doi:10.1029/2011JB008384.

Métivier, L. \& Conrad, C.P., 2008. Body tides of a convecting, laterally heterogeneous, and aspherical earth, J. geophys. Res., 113(B11), B11405, doi:10.1029/2007JB005448.

Morris, S. \& Jackson, I., 2009. Diffusionally assisted grain-boundary sliding and viscoelasticity of polycrystals, J. Mech. Phys. Solids, 57(4), 744-761.

Nowick, A. \& Berry, B., 1972. Anelastic Relaxation in Crystalline Materials, Academic Press.

O'Connell, R.J. \& Budiansky, B., 1978. Measures of dissipation in viscoelastic media, Geophys. Res. Lett., 5(1), 5-8.

Park, J. \& Gilbert, F., 1986. Coupled free oscillations of an aspherical, dissipative, rotating Earth: Galerkin theory, J. geophys. Res., 91(B7), 7241-7260.

Petit, G. \& Luzum, B. (eds)., 2010. IERS Conventions, International Earth Rotation and Reference Service (IERS) Technical Note No. 36.
Qin, C., Zhong, S. \& Wahr, J., 2014. A perturbation method and its application: elastic tidal response of a laterally heterogeneous planet, Geophys. J. Int., 199(2), 631-647.

Raj, R., 1975. Transient behavior of diffusion-induced creep and creep rupture, Metall. Trans. A, 6(8), 1499-1509.

Raj, R. \& Ashby, M.F., 1971. On grain boundary sliding and diffusional creep, Metall. Trans., 2(4), 1113-1127.

Ray, R.D., Eanes, R.J. \& Lemoine, F.G., 2001. Constraints on energy dissipation in the Earth's body tide from satellite tracking and altimetry, Geophys. J. Int., 144(2), 471-480.

Resovsky, J., Trampert, J. \& Van der Hilst, R., 2005. Error bars for the global seismic Q profile, Earth planet. Sci. Lett., 230(3-4), 413-423.

Rivière, A., 2001. 3.5 High Temperature damping, Mater. Sci. Forum, 366368, 268-275.

Sailor, R.V. \& Dziewonski, A.M., 1978. Measurements and interpretation of normal mode attenuation, Geophys. J. Int., 53(3), 559-581.

Shida, T., 1912. On the Elasticity of the Earth and the Earth's Crust, Kyoto Imperial University.

Shito, A., Karato, S.-i. \& Park, J., 2004. Frequency dependence of Q in Earth's upper mantle inferred from continuous spectra of body waves, Geophys. Res. Lett., 31(12), doi:10.1029/2004GL019582.

Sipkin, S.A. \& Jordan, T.H., 1979. Frequency dependence of QScS, Bull. seism. Soc. Am., 69(4), 1055-1079.

Smith, M.L. \& Dahlen, F.A., 1981. The Period and Q of the Chandler wobble, Geophys. J. Int., 64(1), 223-281.

Takei, Y. \& Holtzman, B.K., 2009. Viscous constitutive relations of solidliquid composites in terms of grain boundary contiguity: 2. Compositional model for small melt fractions, J. geophys. Res., 114(B6), B06206, doi:10.1029/2008JB005851.

Tromp, J. \& Dahlen, F.A., 1990. Summation of the Born series for the normal modes of the Earth, Geophys. J. Int., 100(3), 527-533.

Wahr, J. \& Bergen, Z., 1986. The effects of mantle anelasticity on nutations, earth tides, and tidal variations in rotation rate, Geophys. J. Int., 87(2), 633-668.

Wahr, J.M., 1981a. A normal mode expansion for the forced response of a rotating earth, Geophys. J. R. astr. Soc., 64(3), 651-675.

Wahr, J.M., 1981b. Body tides on an elliptical, rotating, elastic and oceanless earth, Geophys. J. R. astr. Soc., 64(3), 677-703.

Wang, R., 1994. Effect of rotation and ellipticity on earth tides, Geophys. J. Int., 117(2), 562-565.

Woodhouse, J.H., 1980. The coupling and attenuation of nearly resonant multiplets in the Earth's free oscillation spectrum, Geophys. J. Int., 61(2), 261-283.

Woodhouse, J.H. \& Dahlen, F.A., 1978. The effect of a general aspherical perturbation on the free oscillations of the Earth, Geophys. J. Int., 53(2), 335-354.

Yang, H.-Y. \& Tromp, J., 2015. Synthetic free-oscillation spectra: an appraisal of various mode-coupling methods, Geophys. J. Int., 203(2), 11791192.

Yuan, L. \& Chao, B.F., 2012. Analysis of tidal signals in surface displacement measured by a dense continuous GPS array, Earth planet. Sci. Lett., 355-356, 255-261.

Yuan, L., Chao, B.F., Ding, X. \& Zhong, P., 2013. The tidal displacement field at Earth's surface determined using global GPS observations, $J$. geophys. Res., 118(5), 2618-2632.

\section{APPENDIX A: MODE EXCITATION BY A TIDAL POTENTIAL}

The tidal potential, $\Psi(\mathbf{r} ; t)$, exerted at a site $\mathbf{r}=[r, \theta, \psi]$ by some celestial body of mass $M_{i}$ located at $\mathbf{r}_{i}=\left[r_{i}(t), \theta_{i}(t), \psi_{i}(t)\right]$ is given by

$\Psi(\mathbf{r} ; t)=-\frac{G M_{i}}{\left\|\mathbf{r}-\mathbf{r}_{i}(t)\right\|}=-\frac{G M_{i}}{d_{i}} \sum_{\ell=0}^{\infty}\left(\frac{r}{d_{i}}\right)^{\ell} P_{\ell}\left(\cos \xi_{i}\right)$,

where $d_{i}=\left\|\mathbf{r}-\mathbf{r}_{i}(t)\right\|$ and the azimuth, $\xi_{i}$, between $\mathbf{r}$ and $\mathbf{r}_{i}$ is

$\hat{\mathbf{r}} \cdot \hat{\mathbf{r}}_{i}=\cos \xi_{i}=\cos \theta \cos \theta_{i}+\sin \theta \sin \theta_{i} \cos \left(\psi-\psi_{i}\right)$. 
$P_{\ell}(x)$ is the Legendre polynomial of degree $\ell$ (or the associated Legendre function of order 0 ). We may express $\Psi$ as

$\Psi(\mathbf{r} ; t)=H_{i}(t) \sum_{\ell=0}^{\infty}\left(\frac{r}{d_{i}}\right)^{\ell} P_{\ell}\left(\cos \xi_{i}\right)$,

where $H_{i}=-G M_{i} / d_{i}(t) . \Psi$ may also be expressed this as in eq. (27), where

$c_{\ell m}=H_{i}\left(\frac{4 \pi}{2 \ell+1}\right) Y_{\ell m}{ }^{*}\left(\theta_{i}, \psi_{i}\right)$

To arrive at eq. (30) from eq. (29), we begin by substituting expressions for $\mathbf{s}_{k}$ (eq. 28) and $\Psi$ (eq. 27) into the following expression

$\mathcal{J}=\sum_{n}^{\infty} \sum_{\ell}^{\infty} \sum_{m}^{\infty} \mathbf{s}_{\ell m}(\mathbf{r}) \int \rho\left(r^{\prime}\right)_{n} \mathbf{s}_{\ell m}{ }^{*}\left(\mathbf{r}^{\prime}\right) \cdot \nabla \Psi_{\ell m}(\mathbf{r} ; t) \mathrm{d} V^{\prime}$

which yields

$$
\begin{aligned}
{ }_{n} \mathcal{J}_{\ell m}= & \left({ }_{n} U_{\ell} Y_{\ell m} \hat{\mathbf{r}}+\frac{{ }_{n} V_{\ell}}{\sqrt{\ell(\ell+1)}} \nabla_{1} Y_{\ell m}\right) \cdot \int \rho\left(\mathbf{r}^{\prime}\right)\left[{ }_{n} U_{\ell} Y_{\ell m}{ }^{*} \hat{\mathbf{r}}+\frac{{ }_{n} V_{\ell}}{\sqrt{\ell(\ell+1)}} \nabla_{1} Y_{\ell m}{ }^{*}\right] . \\
& H_{i}\left(\frac{4 \pi}{2 \ell+1}\right) Y_{\ell m}{ }^{*}\left(\theta_{i}, \psi_{i}\right) \exp \left[i \omega_{T} t\right] \frac{r^{\ell-1}}{a^{\ell}}\left[\ell Y_{\ell m} \hat{\mathbf{r}}+\sqrt{\ell(\ell+1)} \nabla_{1} Y_{\ell m}\right] \mathrm{d} V^{\prime} .
\end{aligned}
$$

We have dropped the eigenfunction dependence on $m$ due to the spherical symmetry of the Earth model. Using the following relations

$\int_{A} Y_{\ell m} \hat{\mathbf{r}} \cdot Y_{\ell^{\prime} m^{\prime}}{ }^{*} \hat{\mathbf{r}} \mathrm{d} A=\delta_{\ell \ell^{\prime}} \delta_{m m^{\prime}}$,

and

$\int_{A} \frac{\left(\nabla_{1} Y_{\ell m}\right) \cdot\left(\nabla_{1} Y_{\ell^{\prime} m^{\prime}}{ }^{*}\right)}{\sqrt{\ell(\ell+1)} \sqrt{\ell^{\prime}\left(\ell^{\prime}+1\right)}} \mathrm{d} A=\delta_{\ell \ell^{\prime}} \delta_{m m^{\prime}}$

where $A$ represents the unit area of a sphere, eq. (A6) simplifies to

${ }_{n} \mathcal{J}_{\ell m}=\left({ }_{n} U_{\ell} Y_{\ell m} \hat{\mathbf{r}}+\frac{{ }_{n} V_{\ell}}{\sqrt{\ell(\ell+1)}} \nabla_{1} Y_{\ell m}\right) \times H_{i}\left(\frac{4 \pi}{2 \ell+1}\right) Y_{\ell m}{ }^{*}\left(\theta_{i}, \psi_{i}\right) \exp \left(i \omega_{T} t\right) \int \rho(r) \frac{r^{\ell-1}}{a^{\ell}}\left[\ell_{n} U_{\ell}+\sqrt{\ell(\ell+1)_{n}} V_{\ell}\right] \mathrm{d} r$

To compute the amplitude of the tidal response, we evaluate $Y_{\ell m}$ at $\left[\theta_{i}, \psi_{i}\right]$. Using the identity

$P_{\ell}\left(\cos \theta_{i}\right)=\left(\frac{4 \pi}{2 \ell+1}\right) \sum_{m=-\ell}^{\ell} Y_{\ell m}\left(\theta_{i}, \psi_{i}\right) Y_{\ell m}{ }^{*}\left(\theta_{i}, \psi_{i}\right)$

yields

${ }_{n} \mathcal{J}_{\ell}=H_{i}\left({ }_{n} U_{\ell} P_{\ell}\left(\cos \theta_{i}\right) \hat{\mathbf{r}}+\frac{{ }_{n} V_{\ell} \nabla_{1} P_{\ell}\left(\cos \theta_{i}\right)}{\sqrt{\ell(\ell+1)}}\right) \int \rho(r) \frac{r^{\ell-1}}{a^{\ell}}\left[\ell_{n} U_{\ell}+\sqrt{\ell(\ell+1)_{n}} V_{\ell}\right] \mathrm{d} r \exp \left[i \omega_{T} t\right]$.

Finally, applying the expression for the tidal potential given by eq. (A3) results in

$$
\begin{aligned}
\mathcal{J}_{\ell}=\sum_{n} & \left\{{ }_{n} U_{\ell}(r) \int \rho(r) \frac{r^{\ell+1}}{a^{\ell}}\left[\ell_{n} U_{\ell}(r)+\sqrt{\ell(\ell+1)_{n}} V_{\ell}(r)\right] \mathrm{d} r \Psi_{\ell}(\mathbf{r}) \hat{\mathbf{r}} \frac{{ }_{n} V_{\ell}(r)}{\sqrt{\ell(\ell+1)}}\right. \\
& \left.\int \rho(r) \frac{r^{\ell+1}}{a^{\ell}}\left[\ell_{n} U_{\ell}(r)+\sqrt{\ell(\ell+1)_{n}} V_{\ell}(r)\right] \mathrm{d} r \nabla_{1} \Psi_{\ell}(\mathbf{r})\right\} \exp \left[i \omega_{T} t\right] .
\end{aligned}
$$

\section{APPENDIX B: EXTRACTING $Q^{-1}$ FROM $h(\omega)$}

To arrive at eq. (56) recall the definition of $\check{Q}^{-1}$ given by eq. (51). To extract the real and imaginary parts of $h(\omega)$ we begin by rationalizing the denominator of eq. (56) where

$\left(\bar{\omega}_{n}{ }^{2}+\varepsilon_{n}{ }^{2}\right)\left(\bar{\omega}_{n}{ }^{2}+\varepsilon_{n}{ }^{2}\right)^{*}=\left(\bar{\omega}_{n}{ }^{2}+\operatorname{Re}\left\{\varepsilon_{n}{ }^{2}\right\}\right)^{2}+\operatorname{Im}\left\{\varepsilon_{n}{ }^{2}\right\}^{2}$.

Ignoring second-order terms involving $\operatorname{Re}\left\{\varepsilon_{n}{ }^{2}\right\}$ (where $\operatorname{Re}\left\{\varepsilon_{n}{ }^{2}\right\} \ll \bar{\omega}_{n}{ }^{2}$ ) we find that

$\left(\bar{\omega}_{n}{ }^{2}+\varepsilon_{n}{ }^{2}\right)\left(\bar{\omega}_{n}{ }^{2}+\varepsilon_{n}{ }^{2}\right)^{*} \approx \bar{\omega}_{n}{ }^{4}+\operatorname{Im}\left\{\varepsilon_{n}{ }^{2}\right\}^{2}$.

Thus,

$h_{k}(\omega)=\frac{1}{g} \chi_{k}\left[\bar{\omega}_{k}^{2}+\operatorname{Re}\left\{\varepsilon_{k}^{2}\right\}+i \operatorname{Im}\left\{\varepsilon_{k}^{2}\right\}\right]$, 
where

$\chi_{k}=\frac{1}{\bar{\omega}_{k}^{4}+\operatorname{Im}\left\{\varepsilon_{k}^{2}\right\}^{2}} \int_{0}^{a} \rho(r) \frac{r^{\ell+1}}{a^{\ell}}\left[\ell_{n} \bar{U}_{\ell}(r)+\sqrt{\ell(\ell+1)_{n}} \bar{V}_{\ell}(r)\right] \mathrm{d} r$.

From the definition of $\varepsilon^{2}$ (eq. 38) and using eq. (11) (ignoring gravity-related terms), we see that

$\operatorname{Re}\left\{\left\langle\overline{\mathbf{s}}_{k}, \delta \mathcal{H}\left(\omega_{T}\right) \overline{\mathbf{s}}_{k}\right\rangle\right\}=\int_{V} \nabla \mathbf{s}_{k}{ }^{*}: \operatorname{Re}\left\{\delta \boldsymbol{\Lambda}\left(\omega_{T}\right)\right\}: \nabla \mathbf{s}_{k} \mathrm{~d} V$,

$\operatorname{Im}\left\{\left\langle\overline{\mathbf{s}}_{k}, \delta \mathcal{H}\left(\omega_{T}\right) \overline{\mathbf{s}}_{k}\right\rangle\right\}=\int_{V} \nabla \mathbf{s}_{k}{ }^{*}: \operatorname{Im}\left\{\delta \boldsymbol{\Lambda}\left(\omega_{T}\right)\right\}: \nabla \mathbf{s}_{k} \mathrm{~d} V$,

where $\operatorname{Im}\{\delta \boldsymbol{\Lambda}(\omega)\}=\operatorname{Im}\{\boldsymbol{\Lambda}(\omega)\}$. 